\title{
Young Ocean Waves Favor the Rapid Intensification of Tropical Cyclones-A Global Observational Analysis ${ }^{\mathscr{O}}$
}

\author{
LIN ZHANG \\ National Central University, Taoyuan City, Taiwan \\ LEO OEY \\ National Central University, Taoyuan City, Taiwan, and Princeton University, Princeton, New Jersey
}

(Manuscript received 20 June 2018, in final form 20 September 2018)

\begin{abstract}
Identifying the condition(s) of how tropical cyclones intensify, in particular rapid intensification, is challenging, because of the complexity of the problem involving internal dynamics, environments, and mutual interactions; yet the benefit to improved forecasts may be rewarding. To make the analysis more tractable, an attempt is made here focusing near the sea surface, by examining 23-yr global observations comprising over 16000 cases of tropical cyclone intensity change, together with upper-ocean features, surface waves, and lowlevel atmospheric moisture convergence. Contrary to the popular misconception, we found no statistically significant evidence that thicker upper-ocean layers and/or warmer temperatures are conducive to rapid intensification. Instead, we found in storms undergoing rapid intensification significantly higher coincidence of low-level moisture convergence and a dimensionless air-sea exchange coefficient closely related to the youth of the surface waves under the storm. This finding is consistent with the previous modeling results, verified here using ensemble experiments, that higher coincidence of moisture and surface fluxes tends to correlate with intensification, through greater precipitation and heat release. The young waves grow to saturation in the right-front quadrant as a result of trapped-wave resonance for a group of Goldilocks cyclones that translate neither too slowly nor too quickly, which $70 \%$ of rapidly intensifying storms belong. Young waves in rapidly intensifying storms also produce relatively less (as percentage of the wind input) Stokes-induced mixing and cooling in the cyclone core. A reinforcing coupling between tropical cyclone wind and waves leading to rapid intensification is proposed.
\end{abstract}

\section{Introduction}

In part because of the complex dynamics involved, forecasts of tropical cyclone intensity have not improved significantly in the past several decades (Rogers et al. 2006; Houze et al. 2007; Rappaport et al. 2009; Gall et al. 2013; Rios-Berrios et al. 2014). Yet understanding and identifying the conditions of how a tropical cyclone changes intensity can lead to improved prediction and storm preparedness, which may save lives and properties. Tropical cyclone intensity depends on various often interrelated factors such as the following:

Supplemental information related to this paper is available at the Journals Online website: https://doi.org/10.1175/MWR-D-180214.s1.

Corresponding author: Leo Oey, lyooey@gmail.com
1) the large-scale environment including the ocean and cyclone translation (Schade and Emanuel 1999; Dunion and Velden 2004; Wang and Wu 2004; Zeng et al. 2007; Lin et al. 2008; Hendricks et al. 2010; Sun and Oey 2015; Sun et al. 2017);

2) the inner-core dynamics (Emanuel 1986; Hendricks et al. 2004; Wang and Wu 2004; Montgomery et al. 2009, 2015);

3) the air-sea energy exchanges (Donelan et al. 2004);

4) the upper-tropospheric outflow and inertial instability (Merrill 1988; Rappin et al. 2011); and

5) their mutual influences (e.g., Wang and Wu 2004; Evans et al. 2010).

The physics of tropical cyclone intensification thus involves a wide range of spatial and temporal scales (Wang and $\mathrm{Wu}$ 2004). The literature may be very broadly grouped into one that examines the influence of the environment [e.g., Hendricks et al. (2010); overviews are given in Evans et al. (2010) and Sun et al. (2017)], 
and the other one that studies internal dynamics (Riehl 1963; Emanuel 1991; Montgomery and Smith 2014). There is, however, a considerable overlap of the internaldynamical and environmental processes, and how a tropical cyclone can change intensity likely depends both on the inner-core dynamics and the environments (Houze et al. 2007; Evans et al. 2010). One proposed mechanism of tropical cyclone intensification by internal dynamics is the wind-induced surface heat exchange (WISHE) (Emanuel 1986; Yano and Emanuel 1991), in which there exists a positive-feedback reinforcement between tropical cyclone winds and wind speed-dependent surface enthalpy fluxes. An alternative proposed mechanism does not depend on the reinforcing feedback (Montgomery et al. 2009); instead, the intensification process is fundamentally nonaxisymmetric and involves the growths of highly localized deep convective structures that amplify the local vorticity, which then form vortical hot towers (VHTs). In both mechanisms, boundary layer processes near the air-sea interface play an important role. Although the wind speed dependence of the surface enthalpy flux is not strictly necessary for sustained intensification, the WISHE mechanism can alter the rate of tropical cyclone intensification [Montgomery and Smith (2014); Zhang and Emanuel (2016, see their Figs. 4 and 5)].

Tropical cyclones that undergo rapid intensity change, defined as the change of maximum 1-min winds at $10-\mathrm{m}$ altitude of more than $15 \mathrm{~m} \mathrm{~s}^{-1}$ in a 24 -h period (Kaplan and DeMaria 2003), are of particular interest to forecasters and researchers. They often develop into extremely powerful and dangerous storms, and attract the attention of the general public, as they should. Recent well-known examples are Hurricane Katrina (2005), Typhoon Haiyan (2013), Hurricane Patricia (2015), and Hurricane Irma (2017). Some studies have suggested that reduced surface friction at high wind speeds (Powell et al. 2003; Donelan et al. 2004; Holthuijsen et al. 2012; Takagaki et al. 2016) may favor rapid intensification (Soloviev et al. 2014, 2017; Donelan 2018), which in turn may explain the observed bimodal distribution of lifetime maximum intensity (Kossin et al. 2013; Lee et al. 2016). Barenblatt et al. (2005) modeled the reduced friction by incorporating spray into the marine boundary layer: the suspension of spray droplets depletes turbulence energy production, making the flow more "laminar," resulting in flow acceleration. Holthuijsen et al. (2012; see also Donelan et al. 2004) observed that as hurricane wind speed increases, large amount of streaks of foam and spray merge into a "white out" over the sea surface, effectively smoothing out the surface roughness. Soloviev et al. (2014) showed that the white out can be caused by the breakup of Kelvin-Helmholtz waves (because in very high wind speeds the pressure drop over the wave overcomes gravity and surface tension), which disrupts the air-sea interface and rips droplets from it to form spume, resulting in drag reduction as short gravity-capillary waves are suppressed. On the other hand, spray may also change the surface enthalpy flux that in turn can modify intensification (Lighthill 1999; Andreas and Emanuel 2001). Soloviev et al. (2014, see their Fig. 4) found that the bimodal distribution of lifetime maximum intensity may also be explained by a similar bimodal distribution of the environmental potential intensity. Kowch and Emanuel (2015) have suggested that rapid intensification may be primarily controlled by random environmental and internal variability. These studies further reiterate the complexity of the tropical cyclone intensity problem: while surface momentum and enthalpy fluxes are important, they alone are probably insufficient in explaining intensity changes.

Asymmetry in the structure of a tropical cyclone plays an important role in determining its intensity (Emanuel 1991; Wang and $\mathrm{Wu}$ 2004). Numerical experiments have shown that an idealized symmetrical vortex in a uniformly rotating Earth (the $f$ plane) can achieve high intensity (Peng et al. 1999). A wavenumber-1 azimuthal asymmetry (i.e., the lowest possible asymmetric Fourier wave) begins to develop in the core of the vortex under more realistic conditions such as the latitudinal variation of Earth's Coriolis parameter (the $\beta$ plane), the storm's translation, and the large-scale steering flow and vertical wind shear. Peng et al. (1999) (see also Frank and Ritchie 2001; Wu and Braun 2004) then found that an azimuthal mismatch between the locations of the maximum turbulent air-sea exchange surface flux and lowlevel moisture flux convergence (MFC) weakens the cyclone. As a working hypothesis, we posit that a high areal coincidence of the surface flux and low-level moisture convergence around the cyclone center favors intensification. (The term "coincidence" is used in a geometric sense, from medieval Latin "coincidere," in the sense of "occupying the same space.") The idea is that larger coincidence would favor intensification through greater precipitation and heat release.

This study attempts to identify intensification condition(s) due to physical processes in the atmosphere and at the sea surface. We seek observational evidence if rapidly intensifying tropical cyclones have higher areal coincidence of surface flux and horizontal moisture convergence in their cores than slower and nonintensifying storms. However, instead of "surface flux," we seek a greater generality by using a dimensionless surface waveinduced air-sea exchange coefficient. The complex intensity-change identification problem is thus reduced to examining two parameters, and their coincidences, which, relatively speaking, can be more easily deduced 
from observations. However, as our analysis and discussion (last section) will indicate, this should not be misunderstood as an oversimplistic view of a complex problem.

It is well established that the ocean's surface waves play an indispensable role in characterizing and modulating the air-sea exchange flux (Andreas and Emanuel 2001; Donelan et al. 2004; Holthuijsen et al. 2012; Soloviev et al. 2014; Hwang and Walsh 2016; Takagaki et al. 2016). To study the coincidence of surface flux and low-level moisture flux convergence, we therefore use observations of tropical cyclone-induced surface waves to estimate the patterns of a dimensionless waveinduced air-sea exchange flux coefficient, and compare them with the patterns of the moisture flux convergence for global tropical cyclones under different states of the intensification process. The global data yield a total of over 16000 cases, from which we may obtain stable composites with a high confidence level. Our analysis will show that rapidly intensifying tropical cyclones have younger surface waves, as well as significantly higher coincidence and larger low-level moisture flux convergence and ocean wave-induced air-sea exchange coefficient in the core of the cyclone than slower-intensifying and weakening cyclones.

For completeness in examining the ocean influence, we also consider the potential impact of warm ocean features on tropical cyclone rapid intensification. Several studies found that in some cases warmer ocean features: thicker upper layers and/or higher surface temperatures may induce rapid intensification (e.g., Shay et al. 2000; Lin et al. 2008). Using the global data, we will show that there is no statistically significant difference in the upper-ocean thickness and/or sea surface temperature under rapidly and nonrapidly intensifying tropical cyclones.

The manuscript is organized as follows. The "methods" section describes in details how the wave and atmospheric observations were prepared, so that the air-sea exchange coefficients and moisture flux convergences could be calculated for each of the 16000 cases of intensity change, and their coincidences and degrees of asymmetry estimated for different intensity-change groups. In the "results" section, we first describe the patterns of the observed air-sea exchange coefficient and moisture flux convergence for different intensity-change groups. We then discuss their significances focusing on distinguishing between the results of rapidly and nonrapidly intensifying tropical cyclones. The section then analyzes the potential effects of warm ocean features on intensity change, and ends with a brief summary of the overall finding and implication. The final section proposes a plausible mechanistic feedback between wave-induced enthalpy flux and intensity change; there, we also discuss how ocean mixing by the Stokes's currents (Stokes 1880) contributes in explaining why young ocean waves could favor the rapid intensification of tropical cyclones.

\section{Methods}

\section{a. Momentum exchange coefficient}

Tropical cyclone winds impart energy to ocean surface waves. In return, surface waves determine air-sea exchanges of momentum and energy by producing roughness and sea spray at the air-sea interface (Lighthill 1999; Donelan et al. 2004; Black et al. 2007; Holthuijsen et al. 2012; Soloviev et al. 2014), which affect cyclone intensity (Riehl 1963; Emanuel 1986, 1991; Lighthill 1999). We estimate air-sea exchange through knowledge of surface wave significant wave height $H_{s}$ obtained from satellite altimeter data in tropical cyclones, as follows.

Excluding the eye region, surface waves under a tropical cyclone cannot in general propagate faster than the strong cyclone's wind; they are constantly under the influence of the strong wind and may be classified as active wind seas. The inverse wave age $\omega_{n}=U_{10} / C_{p}$ generally exceeds one; here $U_{10}$ is the magnitude of wind vector $\mathbf{U}_{10}$ at 10 -m altitude, $C_{p}=(g / \omega)$ is deep-water wave phase speed, $g$ is acceleration due to gravity, and $\omega$ is wave frequency. Hwang and Walsh (2016) (see also Young 2017) show that the dimensionless wave growth functions are then comparable to those in field experiments collected under more ideal quasi-steady fetch-limited conditions (Toba 1972; Donelan et al. 1992; Hwang and Wang 2004; Mellor et al. 2008). We use the wave growth function of Hwang and Walsh (2016), Eq. (1a), to express the air-sea momentum exchange (i.e., wind input to generate surface wave momentum) $M_{t}$, Eq. (1b), in terms of $U_{10}$ and significant wave height $H_{s}$ :

$$
\begin{aligned}
& \eta_{n}=2.94 \times 10^{-3} \omega_{n}^{-3.42} \\
& M_{t}=\alpha_{M} \rho_{a} U_{10}^{2}, \quad \alpha_{M}=2.63 \times 10^{-4} \eta_{n}^{-0.26}, \\
& \eta_{n}=\left[C_{H_{s}} /\left(2 U_{10}\right)\right]^{4}, \quad C_{H_{s}}=\left(g H_{s}\right)^{1 / 2} .
\end{aligned}
$$

Here, $\rho_{a}$ is the air density, $\eta_{n}$ is the dimensionless significant wave height, and $\alpha_{M}$ is the dimensionless momentum exchange coefficient. The $\eta_{n}$ is also the dimensionless spectral wave energy: $\left[\left(g H_{s} / 4\right) / U_{10}^{2}\right]^{2}$ (Longuet-Higgins 1952). Using the wave growth function in Eq. (1a), we obtain

$$
\alpha_{M} \approx 10^{-3} \times\left(U_{10} / C_{H_{s}}\right) \approx 1.9 \times 10^{-3} \omega_{n}^{0.86} .
$$

The momentum exchange coefficient $\alpha_{M}$ is therefore proportional to the ratio of local wind speed to $C_{H_{s}}$, and is directly related to the inverse wave age $\omega_{n}$ : young waves have larger $\alpha_{M}$. Hwang and Walsh (2016) showed 
that the $\alpha_{M}$ is closely related to the surface drag coefficient $C_{D}$, and found good agreements between the two coefficients using observations in Hurricane Bonnie (1998) for wind speeds up to $42 \mathrm{~m} \mathrm{~s}^{-1}$. At high wind $\left(30<U_{10}<50 \mathrm{~m} \mathrm{~s}^{-1}\right)$, the $C_{D}$ has been found to saturate and/or decrease in laboratory and field experiments (Powell et al. 2003; Donelan et al. 2004; Jarosz et al. 2007; Holthuijsen et al. 2012; Takagaki et al. 2012; Soloviev et al. 2014). Donelan et al. (2012; and others) showed that the $C_{D}$ too depends on waves through the Jeffreys's (1925) sheltering coefficient $A_{1}$ modulating the wind energy input $\left(S_{\text {in }}\right)$ to the waves. Using laboratory and field observations, Donelan (2018) deduced that the saturation and/or decrease of $C_{D}$ is due to a Reynolds number-dependent $A_{1}$, which is analogous to the Reynolds number-dependent behavior of form drag for flow past a solid cylinder of fixed diameter (Batchelor 1967, see their section 5.11). The $A_{1}$ would decrease and increase gradually as the Reynolds number increases, and would drop precipitously as the Reynolds number exceeds a critical value. For completeness, we recalculated $A_{1}$, following Donelan (2018) (see the appendix), ${ }^{1}$ and used it in the wind energy input $S_{\text {in }}$ (Hwang and Walsh 2016) to obtain a modified $\alpha_{M}$ (i.e., $\alpha_{\mathrm{MM}}$ ) so that it too is Reynolds number dependent and saturates/ decreases near $U_{10} \approx 30-50 \mathrm{~m} \mathrm{~s}^{-1}$. The coincidence analysis (below) is insensitive to either $\alpha_{M}$, however, and both give very similar results.

Peng et al. (1999) found in their tropical cyclone model that the moisture and heat fluxes in the core have basically the same pattern as the momentum flux. Guided by their results, we therefore also assume that the pattern of momentum flux $M_{t}$ is similar to the pattern of surface fluxes of heat and moisture. However, instead of $M_{t}$, it is more desirable to examine $\alpha_{M}$, which is dimensionless and is much less dependent on the details of the tropical cyclone wind distribution in the core; we use it to present our results.

\section{b. Storm-following maps}

We used 6-hourly tropical cyclone data from the IBTrACS archive (Knapp et al. 2010) from 1992 to 2015 (Fig. 1a). We followed each cyclone and constructed $800 \mathrm{~km} \times 800 \mathrm{~km}$ storm-centered, 6-hourly maps with $10-\mathrm{km}$ grid resolution. The maps were rotated with a positive $y$ axis in the instantaneous direction of the storm, and a positive $x$ axis at $90^{\circ}$ to the right. An example is shown in Fig. $1 \mathrm{~b}$ for such a map centered at the

\footnotetext{
${ }^{1}$ The late Professor Donelan's septic formula, Eq. (13) of Donelan (2018), does not fit his Fig. 5. The corrected formula is given in the appendix.
}

center of Typhoon Haiyan on 7 November 2013. Variables were gridded on these maps and were then composited (i.e., arithmetically averaged) in four 24-h intensity-change $(\Delta V)$ groups: rapid intensification (RI), $\Delta V>15 \mathrm{~m} \mathrm{~s}^{-1}$; slow to medium intensification (SMI), $15 \geq \Delta V>5 \mathrm{~m} \mathrm{~s}^{-1}$; neutral $(\mathrm{N}), 5 \geq \Delta V>-5 \mathrm{~m} \mathrm{~s}^{-1}$; and weakening (W), $-5 \mathrm{~m} \mathrm{~s}^{-1} \geq \Delta V$ (Fig. 1a). Here $\Delta V$ is the change in the peak 1-min wind speeds $V_{m}$ at 10-m altitude. We found that weaker, tropical storms with $V_{m}<33 \mathrm{~m} \mathrm{~s}^{-1}$ generally have a large percentage of older waves (see Figs. S1a-d in the online supplemental material), for which the use of the wave growth function may be problematic. We therefore chose only track points with $V_{m}>33 \mathrm{~m} \mathrm{~s}^{-1}$, which included $98 \%$ of all rapidly intensifying storms. To avoid near-coast regions where intensity changes may be influenced by terrain, we used the ETOPO2 2-min resolution dataset to choose only those tracks over the open ocean where the water depth is deeper than $1000 \mathrm{~m}$.

\section{c. Significant wave height}

The 6-hourly tracks of $H_{s}$ from satellite altimeter (AVISO) were gridded without interpolation onto the above storm-following, storm-centered 6-hourly maps. The satellite $H_{s}$ was checked against NDBC buoy data. These buoys were mostly located near the coast and over the continental shelves and slopes of the United States. The comparison yielded a regression slope $=1.1$ (satellite underestimates) and $r^{2}=0.97$.

\section{d. Wind}

The $10-\mathrm{m}$ wind $\mathbf{U}_{10}$ was calculated using a parametric tropical cyclone model (Holland et al. 2010) using 6-hourly center pressure, location, and $V_{m}$ from the IBTrACS, the radius of maximum wind estimated from Knaff et al. (2007) with the cyclone-moving component added following Jakobsen and Madsen (2004). The tropical cyclone wind was then merged with the crosscalibrated multi-platform (CCMP) reanalysis wind (Atlas et al. 2011) at radial distances $>\sim 350 \mathrm{~km}$ from the cyclone center. The merged wind was then gridded onto the storm-centered maps using bilinear interpolation. The data were checked for self-consistency against observed NDBC buoy winds and maximum wind speeds for tropical cyclones from 1992 to 2015, yielding a regression slope $=1.02$ and $r^{2}=0.93$.

The $\mathbf{U}_{10}$ was bilinearly interpolated onto the stormfollowing maps. Then together with the $H_{s}$, other derived variables such as $\omega_{n}, \alpha_{M}$, etc., from Eqs. (1) and (2) were calculated on the maps.

\section{e. Moisture flux}

Low-level column-integrated moisture flux convergence (MFC) and the column-integrated moisture flux 
(a)

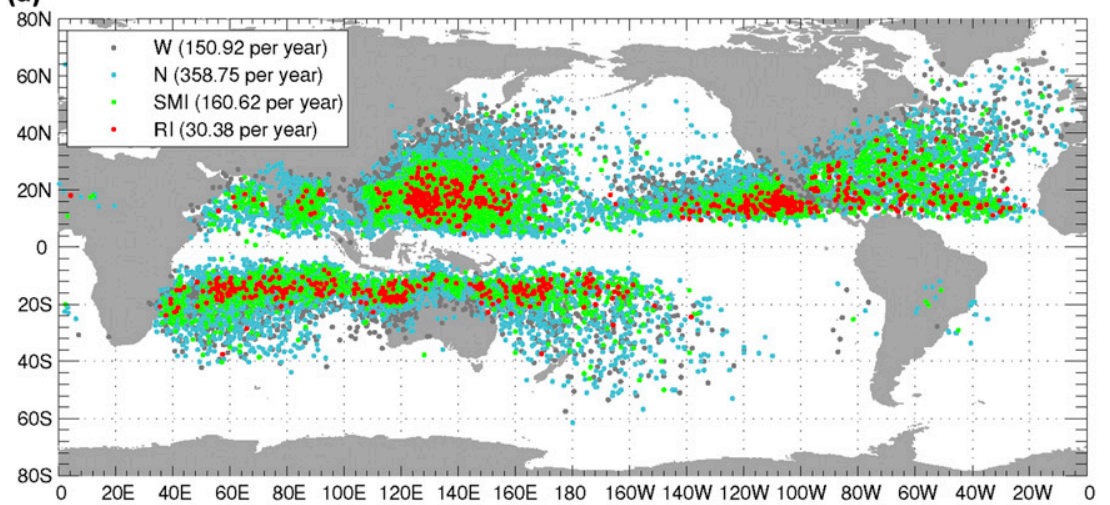

(b)
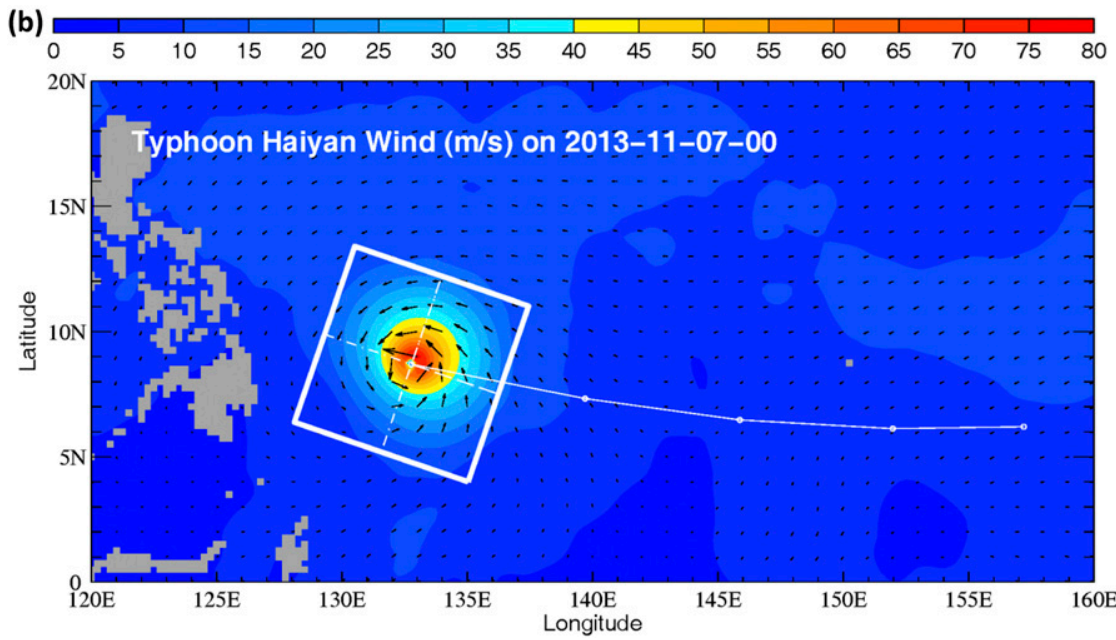

FIG. 1. IBTrACS data from 1992 to 2015. (a) Locations of rapidly intensifying (RI, red), slow to medium intensifying (SMI, green), neutral (N, cyan), and weakening (W, gray) tropical cyclones of category 1 and stronger. Legends show the mean occurrence per year for each case. (b) An example [Typhoon Haiyan wind vector and speed (color shading) on 7 Nov 2013] of a storm-following, storm-centered $800 \mathrm{~km} \times 800 \mathrm{~km}$ box within which wind, waves, and other analyzed parameters such as the moisture flux convergence MFC and air-sea exchange coefficient $\alpha_{M}$ are calculated and composited.

(I $\left.\mathbf{I}_{\text {uq }}\right)$ were computed using wind, specific humidity (calculated from temperature and relative humidity) data from the 6-hourly National Centers for Environmental Prediction Final Operational Global Analysis [(NCEP-FNL) from 2000 to 2015] analysis at $1^{\circ}$ resolution:

$$
\begin{aligned}
\mathrm{MFC} & =-\frac{1}{\rho g} \int_{1000}^{700} \nabla \cdot(\mathbf{u q}) d p, \quad \text { and } \\
\mathbf{I}_{\mathbf{u q}} & =-\frac{1}{\rho g} \int_{1000}^{700} \mathbf{u q} d p .
\end{aligned}
$$

The upper-bound pressure for the integration was $700 \mathrm{hPa}$, and the lower bound was the surface pressure. The $1^{\circ} \times 1^{\circ} \mathrm{NCEP}$ data are too coarse to resolve the tropical cyclone core and the wind magnitude is also underestimated. However, observations have been assimilated in the NCEP data, and we focus only on the asymmetric pattern of the MFC, which is caused primarily by the interaction of the cyclone with the larger-scale environment (Peng et al. 1999). We expect that the MFC pattern and its relative distribution with respect to the pattern of $\alpha_{M}$ are little affected by the magnitude of MFC. The MFC and $\mathbf{I}_{\mathbf{u q}}$ were gridded onto the storm-centered maps using bilinear interpolation.

\section{f. Degree of asymmetry (DOA)}

The degree of asymmetry of the moisture flux $\mathbf{I}_{\mathbf{u q}}$ is $\operatorname{DOA}(\mathrm{MF})=\sum\left(I_{u q a}^{2}+I_{v q a}^{2}\right) / \sum\left(I_{u q}^{2}+I_{v q}^{2}\right)$, subscript " $a$ " indicates the asymmetric component of the flux (i.e., total minus the axis-symmetric part), and the summation is over grid points within the circle of radius $200 \mathrm{~km}$ about the storm center. The DOAs for scalars $\alpha_{M}$ and 
MFC [i.e., $\operatorname{DOA}\left(\alpha_{M}\right)$ and $\operatorname{DOA}(\mathrm{MFC})$ ] are similarly defined by partitioning each into asymmetric and axissymmetric components. The DOA(MF) and DOA(MFC) are very similar; only $\mathrm{DOA}(\mathrm{MF})$ is shown below.

\section{g. Composites}

We calculated composites of variables for all selected tracks in the global ocean (Fig. 1a), and separately also in each of the six tropical cyclone basins, as well as the Northern and Southern Hemispheric basins. Southern Hemispheric storms were mapped into Northern Hemispheric-equivalent storms by reflecting the field in the storm-following maps into the $y$ axis: $\operatorname{Field}(x, \ldots)=\operatorname{Field}(-x, \ldots)$. Since rapid intensification occurs mostly near the tropics (Fig. 1a), the atmospheric and SST environments are different from those at higher latitudes where many nonrapidly intensifying changes could occur. It is necessary therefore to check for possible biases in the composites due to the different geographic environments. We therefore repeated the composite calculations of moisture flux vectors and convergences using only those storm tracks within the $30^{\circ} \mathrm{S}-30^{\circ} \mathrm{N}$ latitude band. The wave-related composites (e.g., $\omega_{n}$ ) are unchanged however. (Tests using waves also within $30^{\circ} \mathrm{S}-30^{\circ} \mathrm{N}$ yielded virtually identical $\omega_{n}$ patterns.) Standard statistical tests (e.g., Press et al. 1992) were applied to calculate the significances of the composites and differences. The nonparametric WilcoxMann-Whitney test (Chu 2002) was also used to calculate the significance of differences, yielding similar results. Because of the relatively large samples used for global and individual basins, except the north Indian basin, the significance tests were well satisfied at the $95 \%$ confidence level.

\section{h. Ensemble model experiments}

As stated previously in the introduction, an objective of the present study is to use observations to test the hypothesis that high areal coincidence of the surface flux and low-level moisture convergence around the tropical cyclone center favors intensification (Peng et al. 1999). Peng et al. (1999) conducted single-member experiments. Before presenting the results of the observational analyses, it is desirable to place the hypothesis on a statistically firmer ground. We therefore repeated their $\beta$-plane model experiment of the translation and intensification of an initial vortex; but instead of one experiment, we conducted a suite of experiments with the initial vortex placed at different tropical latitudes, with the model initial fields randomly perturbed, and with different boundary layer and microphysics schemes (see supplemental material for more information). The experiments yielded different cyclones with different trajectories and translation speeds, and over 120 samples of intensity change $\delta V$. Together with the corresponding surface fluxes and MFCs, the change in their coincidence, $\delta$ (Coincidence), was calculated and regressed against $\delta V$ (see Fig. S2). There is a close $\left(r^{2} \approx 0.65\right)$, nearly linear relation with positive slope between the two variables: higher coincidences tend to be associated with stronger intensifications, consistent with the Peng's hypothesis.

\section{Results}

\section{a. Patterns and coincidences of $\alpha_{M}$ and MFC}

Excluding the eye region, the RI group (Fig. 2a) has the largest momentum exchange coefficient $\alpha_{M}$ and therefore youngest and most active waves [please see Eq. (2) showing $\alpha_{M}$ nearly linearly related to the inverse wave age $\omega_{n}$ ]. The large $\alpha_{M}$ wraps clockwise around the storm's center, nearly reaching the left-front quadrant, thus making the $\alpha_{M}$ pattern nearly symmetrical. By contrast, young waves in the weakening (W) group (Fig. 2d) are mostly confined to the right side, while older waves may be seen in the left-front quadrant. The SMI (Fig. 2b) and N (Fig. 2c) groups have intermediate $\alpha_{M}$ patterns. All the groups display a common feature that the $\alpha_{M}$ is the largest and most widespread in the right-back quadrant, in agreement with the field measurements and modeling that also show youngest waves in that quadrant (Holthuijsen et al. 2012; Hwang and Walsh 2016). The asymmetric moisture flux enters in the back and exits in the front "through" the cyclone generally in the direction of the translation; it contributes to an MFC that is "front heavy" (Fiorino and Elsberry 1989; Peng et al. 1999). The RI group has the most symmetrical MFC pattern (Fig. 2a), with the region of large MFC shifted toward the right-front quadrant. By contrast, the other three non-RI groups (Figs. 2b-d) show a more widespread asymmetric pattern with larger MFC tilted toward the left-front quadrant. For these non-RI groups, the left-front MFC asymmetry is therefore azimuthally $180^{\circ}$ out of phase with their right-back $\alpha_{M}$ asymmetry, while the phase shift for the RI group is less. These MFC and $\alpha_{M}$ patterns generally hold also for individual basins (Figs. S3-S8) and Northern and Southern Hemispheric composites (Figs. S9-S10); the exceptions are in the north Indian and the South Pacific basins (Figs. S6-S7) where the samples are too few (Fig. S12). The composite for storms within the $30^{\circ} \mathrm{S}-$ $30^{\circ} \mathrm{N}$ latitude band show very similar patterns (Fig. S11). Because of the large sample size, the composite results are relatively insensitive to the different geographic locations of the storms.

We quantify the extent with which the $\alpha_{M}$ and MFC coincide, as shown in Figs. 2e-h. Positive coincidence 

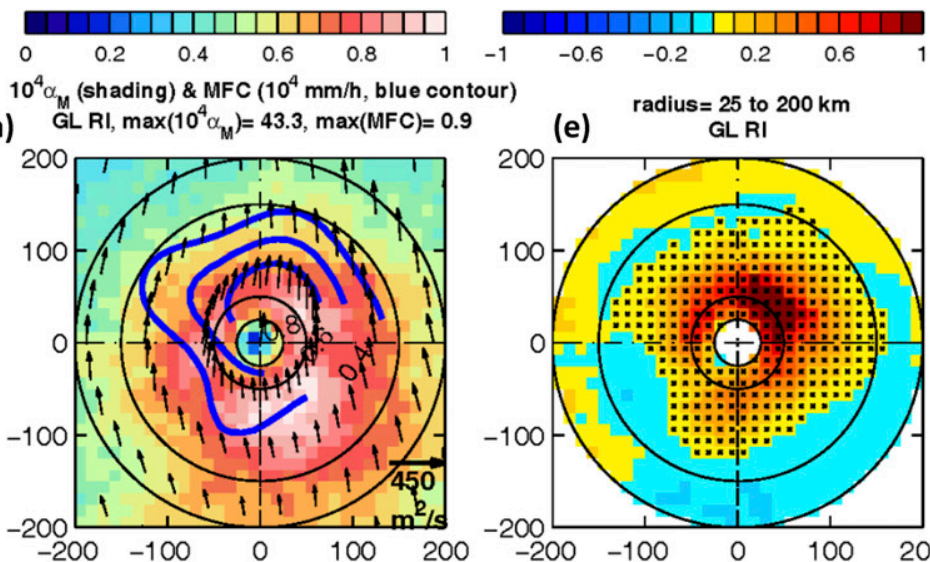

(a) $\mathrm{MLRI} \max \left(10^{4}, 13.3, \max (\mathrm{MFC})=0.9\right.$

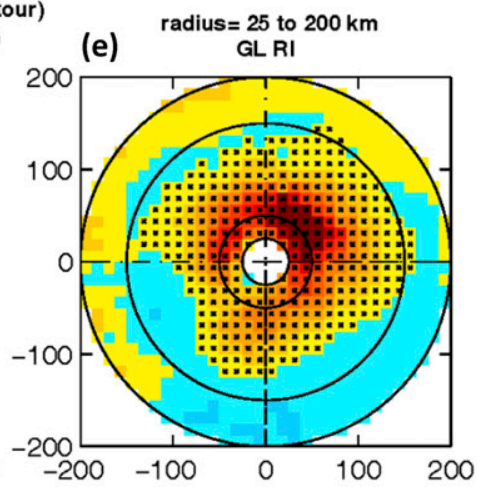

(b) GL SMI, $\max \left(10^{4} \alpha_{M}\right)=39.1, \max (M F C)=0.6$
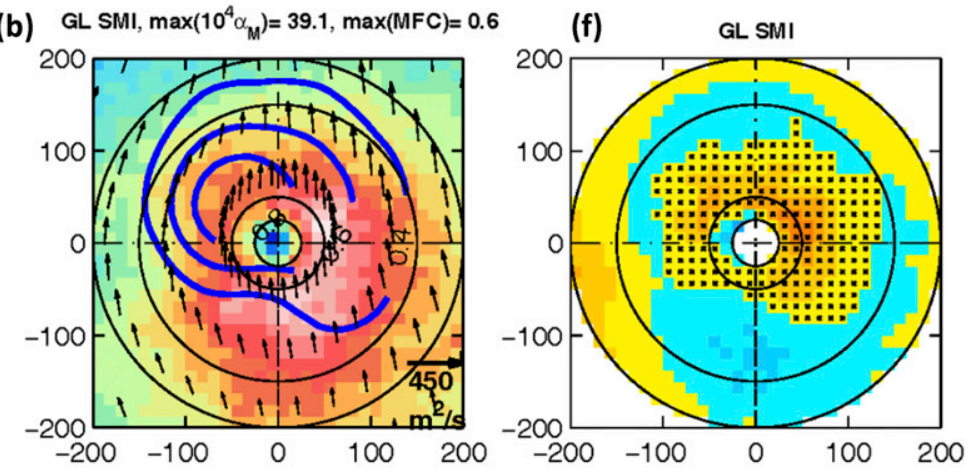

(c)
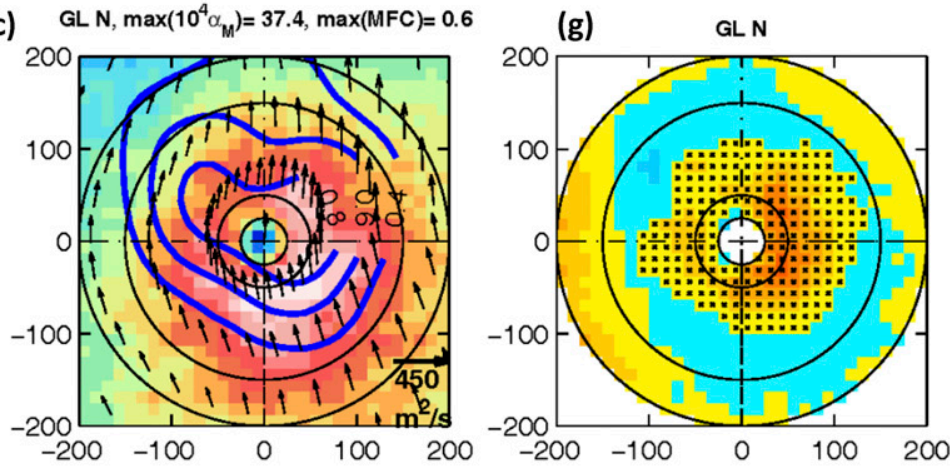

(d)
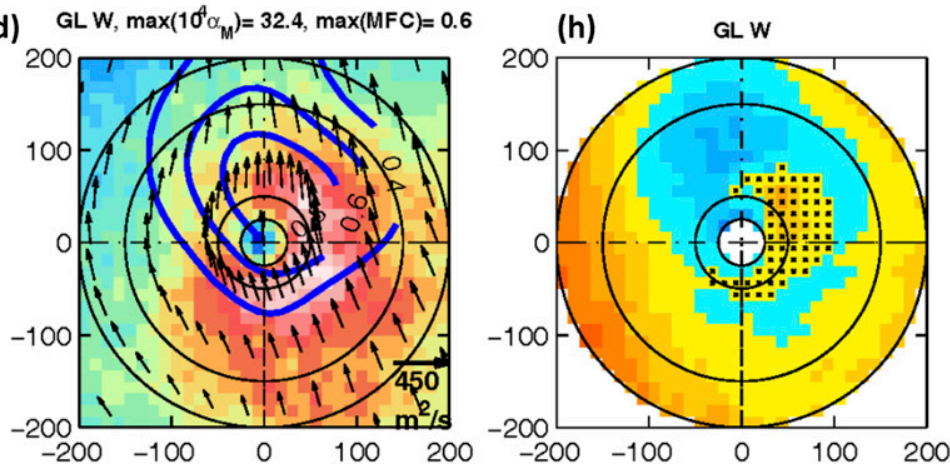

FIG. 2. (a)-(d) Global composites of $\alpha_{M}$ (shading) and MFC (blue contours) each normalized by their maxima shown across the top, and the asymmetric component of the moisture flux (vectors) for each of the four $\Delta V$ groups: RI, SMI, N, and W; values $>0.3$ are significant at the $95 \%$ confidence level. (e)-(h) Corresponding coincidence maps $=$ product of anomalies of $\alpha_{M}$ and MFC; stippling shows positive points where both anomalies are positive. 
points indicate where strong surface and moisture convergence fluxes coincide, while negative coincidence indicates where they are out of phase. The former favors storm intensification while the latter does not (Peng et al. 1999; Wu and Braun 2004). Both the MFC (blue contours on Fig. 2, left column) and $\alpha_{M}$ (color shading) contribute to the coincidence patterns. For the RI (Figs. 2a,e) and W tropical cyclone groups (Figs. 2d,h), the largest $\alpha_{M}$ is on the right-back quadrant (Figs. 2a,d). But because the largest MFC leans toward the rightfront quadrant where the $\alpha_{M}$ while not the largest is quite substantial, the resulting coincidence is controlled more by MFC and therefore its positive region also leans more to the right-front quadrant (Figs. 2e,h). The distinguishing difference between the "RI" and "W" groups is that the former (Figs. 2a,e) has nearly symmetrical MFC and $\alpha_{M}$ (Fig. 2a), thus making their positive coincidence not only larger (as mentioned above), but also more symmetrical (Fig. 2e). For the SMI (Figs. 2b,f) and $\mathrm{N}$ groups (Figs. 2c,g), both the MFC and $\alpha_{M}$ contribute, but the $\alpha_{M}$ has a slightly stronger control over their coincidence patterns.

We summarize these results by plotting the summed positive coincidence (COIN) and the degrees of asymmetry for moisture flux $\mathrm{DOA}(\mathrm{MF})$ and $\alpha_{M} \operatorname{DOA}\left(\alpha_{M}\right)$ (Fig. 3; see Fig. S13 for the corresponding plots for individual basins). For the RI group, the positive coincidence is distributed nearly symmetrically around the core of the storm (Fig. 2e), and the corresponding COIN is significantly higher than the other three non-RI groups (Fig. 3). By contrast, the $\mathrm{W}$ group (Fig. 2h) shows large areas of negative coincidence in the left-front quadrant. The SMI and $\mathrm{N}$ groups have intermediate distributions (Figs. 2f,g). The summed positive coincidences for these two groups are nearly indistinguishable from each other but both remain significantly larger than the COIN of the W group (Fig. 3). For the degrees of asymmetry, both $\operatorname{DOA}\left(\alpha_{M}\right)$ and DOA(MF), are smallest for the RI group, and are significantly smaller than those for the $\mathrm{W}$ group. The analyses for coincidences and degrees of asymmetry for storms confined within the $30^{\circ} \mathrm{S}-30^{\circ} \mathrm{N}$ latitude band show similar stark differences between RI and non-RI groups (Fig. S14). However, the DOAs for the RI group are generally indistinguishable from those of the SMI and $\mathrm{N}$ groups. These results suggest that the coincidence of moisture flux convergence and $\alpha_{M}$ is a more relevant determinant than the axis-symmetry properties in distinguishing rapidly from non-RI tropical cyclones.

We repeated the above analyses (Figs. 2 and 3) to account for a Reynolds number-dependent $\alpha_{M}$ (i.e., $\alpha_{\mathrm{MM}}$ ) such that its value peaks at $U_{10} \approx 30-50 \mathrm{~m} \mathrm{~s}^{-1}$ and then decreases at higher wind speeds (see the methods section and the appendix). (Maps of $\alpha_{\mathrm{MM}}$ and MFC and their coincidences, as

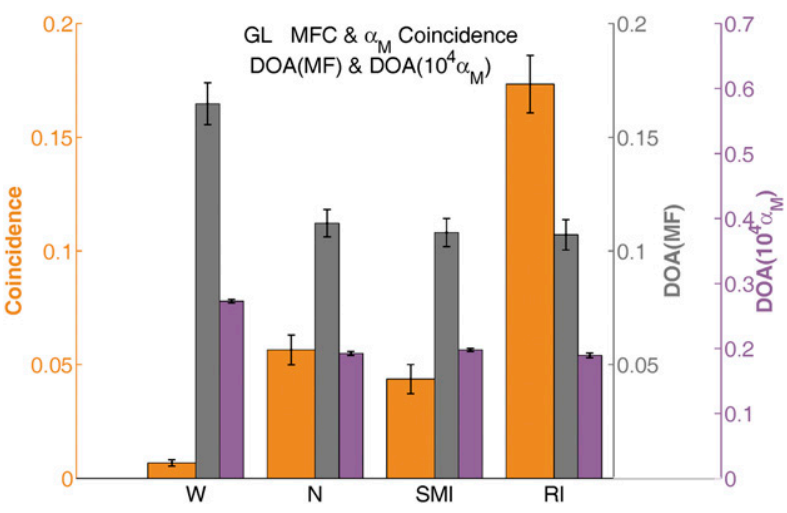

FIG. 3. Summed positive coincidence COIN of MFC and $\alpha_{M}$ (gold bars), the degree of asymmetries of the moisture flux [DOA(MF), gray bars], and $\alpha_{M}$ [DOA $\left(\alpha_{M}\right)$, purple bars] for the four $\Delta V$ groups: RI, SMI, N, and W for the global ocean. Error bars show \pm 1 standard deviation from the mean for that group. The COIN for each group is arbitrarily normalized by the sum (same value for all four groups) of grid points within a $200-\mathrm{km}$ radius surrounding the cyclone.

well as the corresponding summary bar plots are shown in Figs. S15 and S16.) As may be expected, the $\alpha_{\mathrm{MM}}$ values are generally smaller than the $\alpha_{M} \mathrm{~S}$ and the corresponding coincidences are weaker for all intensity change groups, but the patterns are very similar (cf. Figs. 2 and S15). As a result, the contrast in COIN between the RI group and other, non$\mathrm{RI}$ intensity-change groups, while slightly weaker, remains highly significant (cf. Figs. 3 and S16).

\section{$b$. The potential influence of warm ocean features on intensity change}

Previous studies have noted that some tropical cyclones intensified as they passed over warm ocean features (e.g., eddies with diameters of a few hundred kilometers with deepened $26^{\circ} \mathrm{C}$ isotherm in the ocean's upper layer). Shay et al. (2000) observed that Hurricane Opal (1995) rapidly intensified when passing over a warm-core ring in the Gulf of Mexico. Lin et al. (2008) noted eight cases of intensification, from 1993 to 2005, when typhoons passed over warm features in the western North Pacific. Sun et al. (2017) found some correspondence between intensification and warm ocean features for the western North Pacific typhoons, but only obtained high and significant correlations after incorporating other parameters, such as the maximum potential intensity and the wind shear. Here we wish to check if the higher COIN identified above for rapid intensification could also have occurred when the tropical cyclones passed over warm ocean features. Specifically, we ask the following question: Are rapid intensification events more likely to occur, compared to slower intensification events, when tropical cyclones pass over warm ocean features? To answer this question, we 
compared the depth of the ocean upper layer and the sea surface temperature at locations where RI occurred against the same ocean variables when SMI and/or $\mathrm{N}$ intensification occurred. The answer would then be "YES" if the difference (RI minus SMI or RI minus N) is statistically significantly positive; otherwise it is "NO."

As before, we again followed each storm, but this time recorded the sea surface height anomaly (SSHA; http:// www.aviso.altimetry.fr/en/data.html) from satellite altimetry data under the storm. We use the depth of the $26^{\circ} \mathrm{C}$ isotherm, $Z_{26}$, as a measure of the upper-ocean depth (e.g., Goni et al. 1996; Shay et al. 2000; Lin et al. 2008; Sun et al. 2017). Using the reduced gravity approximation, changes in the depth of the $26^{\circ} \mathrm{C}$ isotherm, $Z_{26}^{\prime}$, due to, for example, eddies, can be related to $\eta^{\prime}=$ SSHA: $Z_{26}^{\prime} \approx \eta^{\prime} \rho_{0} / \Delta \rho$ (Goni et al. 1996), where $\rho_{0}$ is a reference (seawater) density and $\Delta \rho$ is the density difference between the upper and lower layer, from the WOA climatology (Stephens et al. 2001). Since $\Delta \rho / \rho_{0}$ varies much slower than $\eta^{\prime}$ both spatially and temporally, $Z_{26}^{\prime} \sim \eta^{\prime}$. This formulation was used by Shay et al. (2000), Lin et al. (2008), Sun et al. (2017), and others; as pointed out by these authors, larger (smaller) SSHAs generally collocate well with thicker (thinner) upperocean warm layers. Note that the ocean heat content correlates well with the $26^{\circ} \mathrm{C}$ isotherm depth (e.g., Oey et al. 2007). Two methods were used to record the resulting SSHA "seen" by the moving storm. The first method simply recorded the SSHA averaged over a small circle of radius $50 \mathrm{~km}$ around the storm center. The second, more stringent method additionally removed a spatially slowly varying SSHA, taken as the averaged SSHA over a 500-km-radius circle around the moving cyclone center, and then averaged the resulting residual or relative SSHA over the $50-\mathrm{km}$ circular area. Similar analyses were applied to the sea surface temperature (SST; https://www.ghrsst.org/). (More details are provided in the supplemental material.) We then compared the SSHA and SST under the storms that underwent RI against the SSHA and SST under the storms that underwent SMI and $\mathrm{N}$ intensifications, by displaying their differences on a $5^{\circ} \times 5^{\circ}$ grid over the global ocean (Figs. 4a,b). The means and standard deviations of these differences for individual and global ocean basins are given as bar plots in Figs. 4c,d. (For completeness, the differences between the RI and $\mathrm{W}$ cases are also shown in Figs. 4c,d). [The result from the less stringent method 1 (i.e., more favorable to the argument of a connection between SSHA/SST and intensification) is shown in Fig. 4, while the corresponding result from the more stringent method 2 is shown in Fig. S17.] Positive (negative) differences indicate that, on average, the RI storms passed over warmer (cooler) features than the
SMI and N storms. If RI occurred consistently more frequently over warmer eddies compared to SMI and N intensifications, the plots in Figs. 4a,b would then show mostly "warm colored" grids that are also statistically significant. Globally, this is only nearly (i.e., "warm" but insignificant) true for the RI storms in the eastern Pacific for the SSHA (Figs. 4a,c), and additionally in the north and south Indian Oceans for SST (Figs. 4b,d). Indeed, the numbers of negative and positive grids are nearly the same (see the legends in Figs. 4a,b). Over most of the global ocean, it is therefore nearly equally likely for all three intensification groups RI, SMI, and N to occur, regardless of whether or not the storm passes over a warm eddy. The global means for "RI minus (SMI+N)" and "RI minus W" SSHAs are in fact not significant (Fig. 4c; "significant" in the sense of mean exceeding plus or minus one standard deviation). The "RI minus W" SSTs (Fig. 4d, red bars) are significantly positive, however, except for the eastern Pacific and north Indian Oceans. However, that is to be expected as cooler SST can more likely weaken a storm. Similar inferences are obtained using the more stringent method 2 (see Fig. S17 and descriptions). It is interesting that, comparing Fig. 4 with the corresponding plots for method 2 (in Fig. S17), many of the warm-colored grids in the former plots had either become "less warm" or had turned "cool" in the latter plots, which suggests that some of the warm grids in Fig. 4 were induced by large-scale warming that has less to do with localized warmer ocean features.

In conclusion, rapid intensification is neither more nor less likely to occur over ocean warm features than other, less-rapid intensifications. We have thus precluded the possibility that the significantly high COIN identified for rapidly intensifying tropical cyclones (Fig. 3) was due to some happenstance when the analyzed storms passed over ocean warm eddies. Following the logical steps used in our analysis, that began with the four intensity groups of tropical cyclones and ended with COIN, one may write the following:

$$
\mathrm{RI} \Rightarrow \text { high COIN }
$$

where " $\Rightarrow$ " denotes the logical symbol "if ... then." While this observational analysis result does not constitute an absolute mathematical truth, the significantly high COIN for the RI group compared to other intensitychange groups suggests that high COIN may likely be a necessary condition (one of several) for a tropical cyclone to rapidly intensify.

\section{Summary and discussion}

We have shown from multidecadal global observations that the coincidence (COIN) of the atmospheric 
(a)

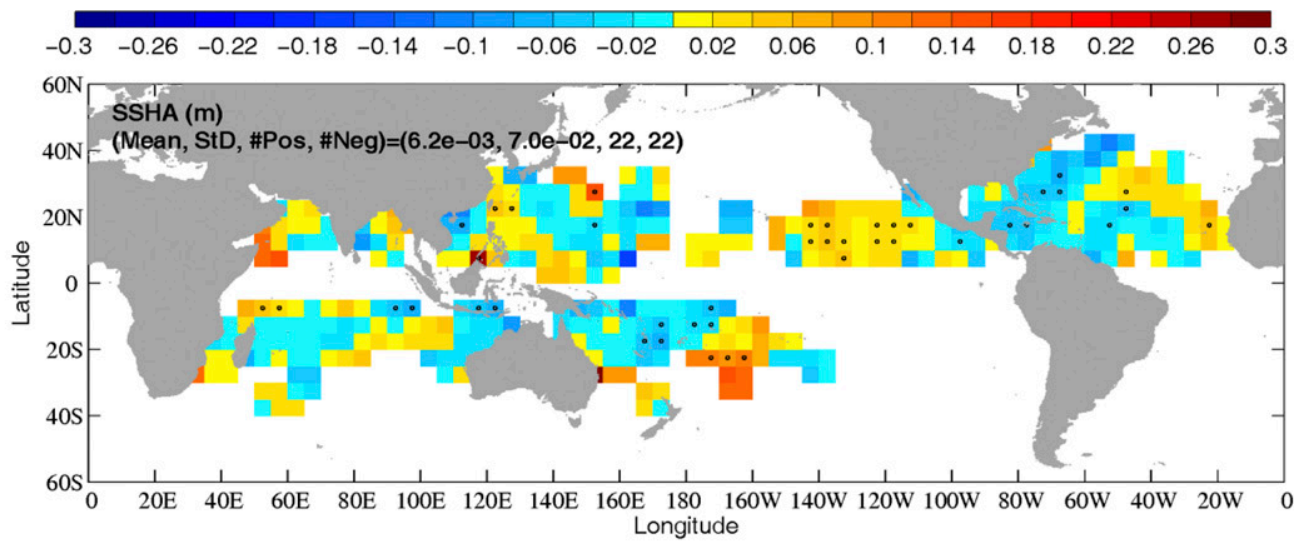

(b)
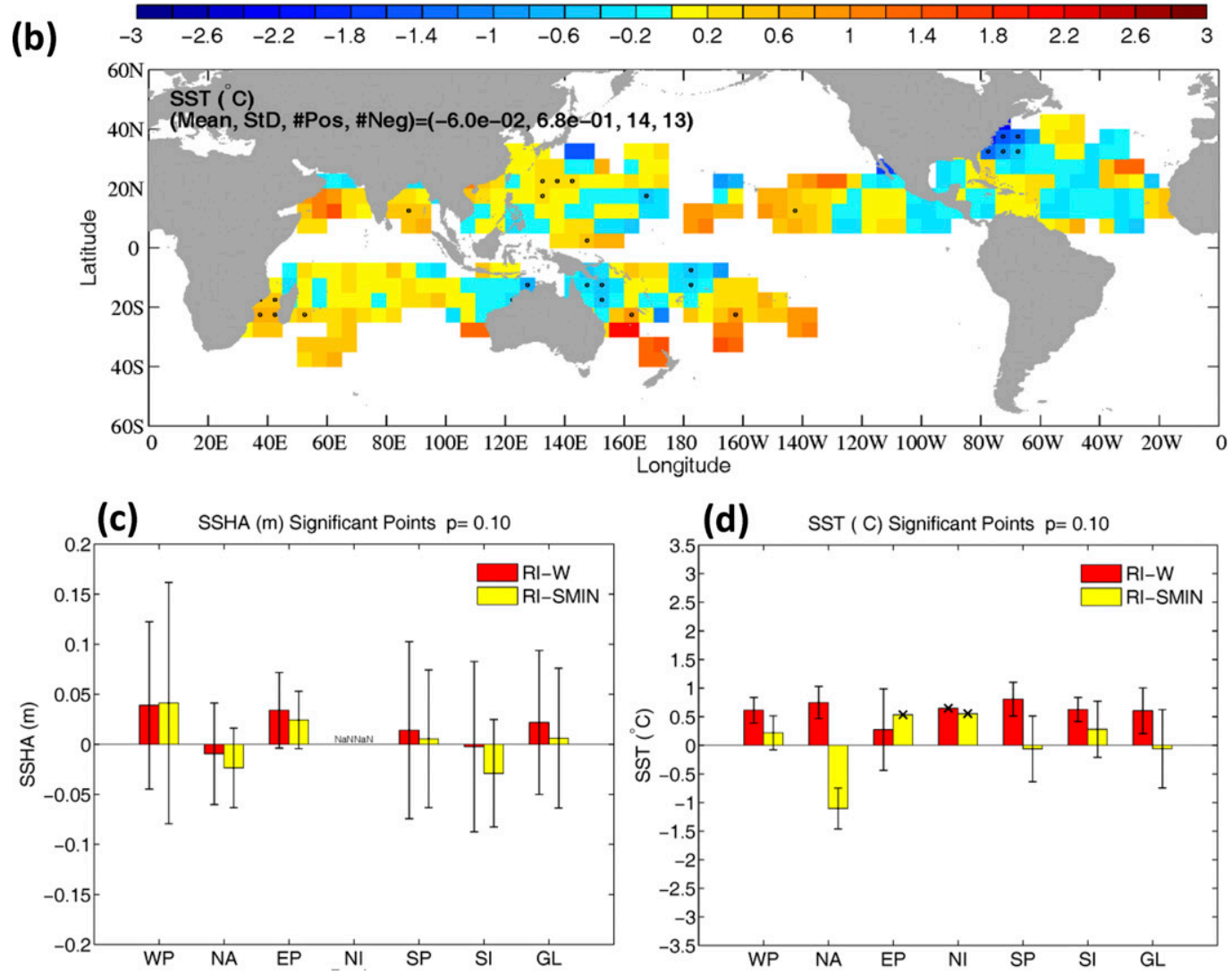

FIG. 4. The RI minus "SMI+N" (a) SSHA and (b) SST; dots indicate significant points at the $90 \%$ confidence level according to the Wilcox-Mann-Whitney test. Legends show global mean, standard deviation (sd), and numbers of positive and negative significant grids. (c),(d) The means (bars) and sd (lines) for individual and global ocean basins, for SSHA and SST, for RI minus W (red) and RI minus "SMI+N" (yellow) storms, averaged from grid points where they are significant [e.g., in (a) and (b) for RI minus "SMI+N"]. The $\times$ s in (d) for the eastern Pacific and north Indian Oceans indicate that the "means" were only for ONE value (i.e., sd $=\infty$ ), and the NaNs in (c) for the north Indian Ocean indicate no significant points.

low-level moisture flux convergence (MFC) and oceanic wave-induced air-sea exchange coefficient $\left(\alpha_{M}\right)$ in the core of a tropical cyclone can serve to differentiate rapid from non-rapid intensity change of the storm. In non-rapidly intensifying storms, the asymmetric component of the MFC is "front heavy" with larger values most likely found in the front of the moving cyclone, while active young waves that contribute to the large $\alpha_{M}$ generally reside in the back. By contrast, in rapidly intensifying storms, the MFC 
becomes more symmetrical; at the same time, young waves appear in the right-front quadrant. These changes in the patterns of the $\mathrm{MFC}$ and $\alpha_{M}$ result in their strong coincidence. Our finding is consistent with the model results of Peng et al. (1999), which we also verified through ensemble experiments, that COIN and intensity change $\Delta V$ are positively correlated. The physical basis, given by Peng et al. (1999), among others, is that an intensifying cyclone tends to be associated with a small wavenumber-1 asymmetry that allows a more symmetric distribution of the surface fluxes and low-level moisture convergence around the center, while a mismatch between the surface flux maximum and the lateral moisture convergence reduces precipitation and prevents intensification.

To understand the high COIN associated with a rapid intensity change, it is therefore necessary to explain why more young waves shift into the rightfront quadrant. The shifting pattern was previously described in the plot for $\alpha_{M} \sim \omega_{n}^{0.86}$ (Figs. 2a-d), but is also clear from the plot of the inverse wave age $\omega_{n}$ itself (see Fig. S18). Suppose a tropical cyclone, of relatively weak or moderate intensity (tropical storm or category 1 ), intensifies (as indicated by the $U_{10}$ "uparrow" symbol near the 7 o'clock position of the clockface in Fig. 5). Finite waves $\left(H_{s} \approx 2 \mathrm{~m}\right.$ or more) grow according to the Jeffreys's (1925) idea that the sheltering of the wave troughs due to flow separation at the wave crests gives rise to form drag, transferring energy from wind to wave (Donelan et al. 2012) (Fig. 5, near the 4 o'clock position). Assuming that the tropical cyclone translates at a speed $U_{h}$ that is near the wave group speed $C_{g}$ (to be shown below), resonant trapped-fetch waves grow to large amplitudes on the right-front side of the cyclone (Moon et al. 2003; Bowyer and MacAfee 2005). As the wind speed $U_{10}$ increases, the inverse wave age $\omega_{n} \approx 0.47 \times\left[U_{10} /\left(g H_{s}\right)^{1 / 2}\right]^{1.2}$ [see Eq. (2)], which is above one, increases further with $U_{10}$ if $U_{10}$ varies like $H_{s}^{k}, k>1 / 2$ (Fig. 5, near the 3 o'clock position). Figure 6 a graphs the probabilities of tropical cyclones in three $U_{h}$ categories: slow, medium, and fast, in bins of the intensity-change $\Delta V$. Figure 6b shows the $C_{g}$ as a function of $U_{10}$ and $H_{s}$, derived by Zhang and Oey (2018), and multiplied by $\cos \left(35^{\circ}\right)$ to adjust for an average misalignment angle of $35^{\circ}$ between waves and wind, the latter being aligned on average with the tropical cyclone's translation on the right side of the storm (Moon et al. 2003; Zhang and Oey 2018). Figures 6c and 6d show the 50th and 90th percentile $H_{s}$ binned according to the maximum wind speed $V_{m}$, in the western Pacific and North Atlantic. (There were insufficient samples in the other basins to derive meaningful statistics.) Figure $6 a$

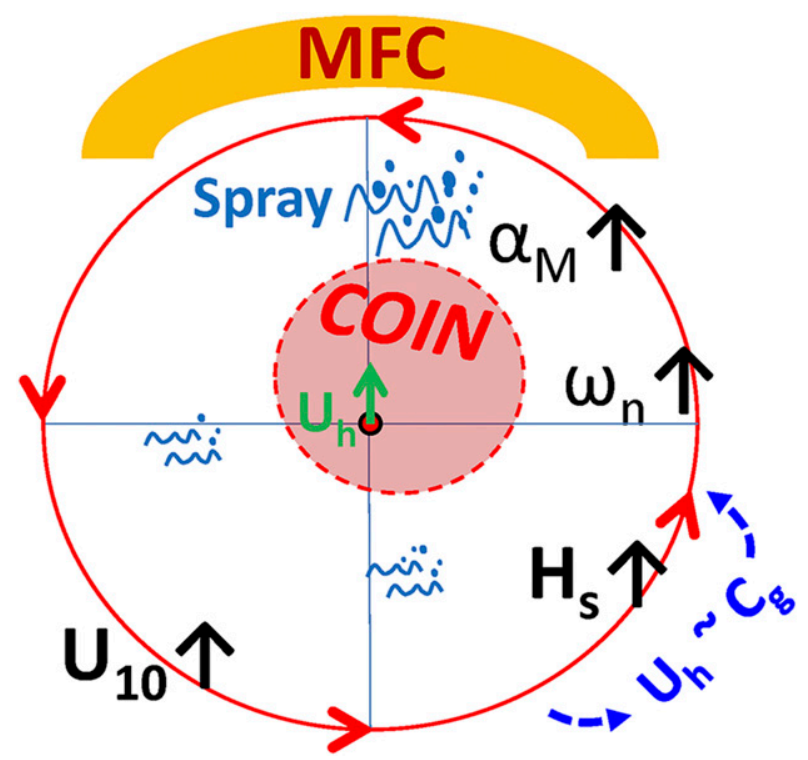

FIG. 5. A sketch of the positive reinforcing loop (big red circle, like a clock, with anticlockwise-directed red arrows indicating positive reinforcement of the physical processes) between tropical cyclone wind and waves that favors rapid intensification as discussed in the text. The tropical cyclone wind is represented by $U_{10}$, wave height is represented by $H_{s}, U_{h}$ is the cyclone's translation speed, $C_{g}$ is the wave's group velocity, $\omega_{n}$ is the inverse wave age, $\alpha_{M}$ is the wave-induced air-sea exchange coefficient, MFC is the moisture flux convergence (anomaly), and COIN is the coincidence between MFC and $\alpha_{M}$. The up arrow $\uparrow$ indicates an increase of the variable preceding it. The pinkshaded dashed circle indicates the area of increased COIN anomaly as a result of the "spilling" of young waves into the right-front quadrant. Effects of the Goldilocks translation speeds, $U_{h} \sim C_{g}$, and the spray droplets due to breaking waves, both favoring intensification, are discussed in the text.

shows that in the rapid intensity change $\Delta V>15 \mathrm{~m} \mathrm{~s}^{-1}$ (i.e., RI) range, the so-called "Goldilocks tropical cyclones" that translate neither too slowly nor too quickly at the medium speeds $U_{h}$ between 3 and $7 \mathrm{~m} \mathrm{~s}^{-1}$, significantly outnumber $(70 \%)$ both the slow $\left(U_{h}<3 \mathrm{~m} \mathrm{~s}^{-1}\right.$; $16 \%)$ and fast $\left(U_{h}>7 \mathrm{~m} \mathrm{~s}^{-1} ; 14 \%\right)$ storms. $^{2}$ In general, the Goldilocks cyclones have a higher probability of intensifying than weakening (the green line slopes up with $\Delta V$ ), while the fast storms have a higher probability of weakening (the red line slopes down), and the slow storms have nearly equal probabilities of intensifying and weakening (the blue line remains mostly flat). Figure $6 \mathrm{~b}$ shows that in the range of $H_{s}$

\footnotetext{
${ }^{2}$ Zeng et al. (2007) had previously noted that western Pacific cyclones moving at the medium-translating speeds are more conducive to intensification. No direct explanation was given; although they suggested that slow storms produce upper-ocean mixing that cools the SST, while fast storms produce large asymmetric structure, both of which are not conducive to intensification.
} 
(a)
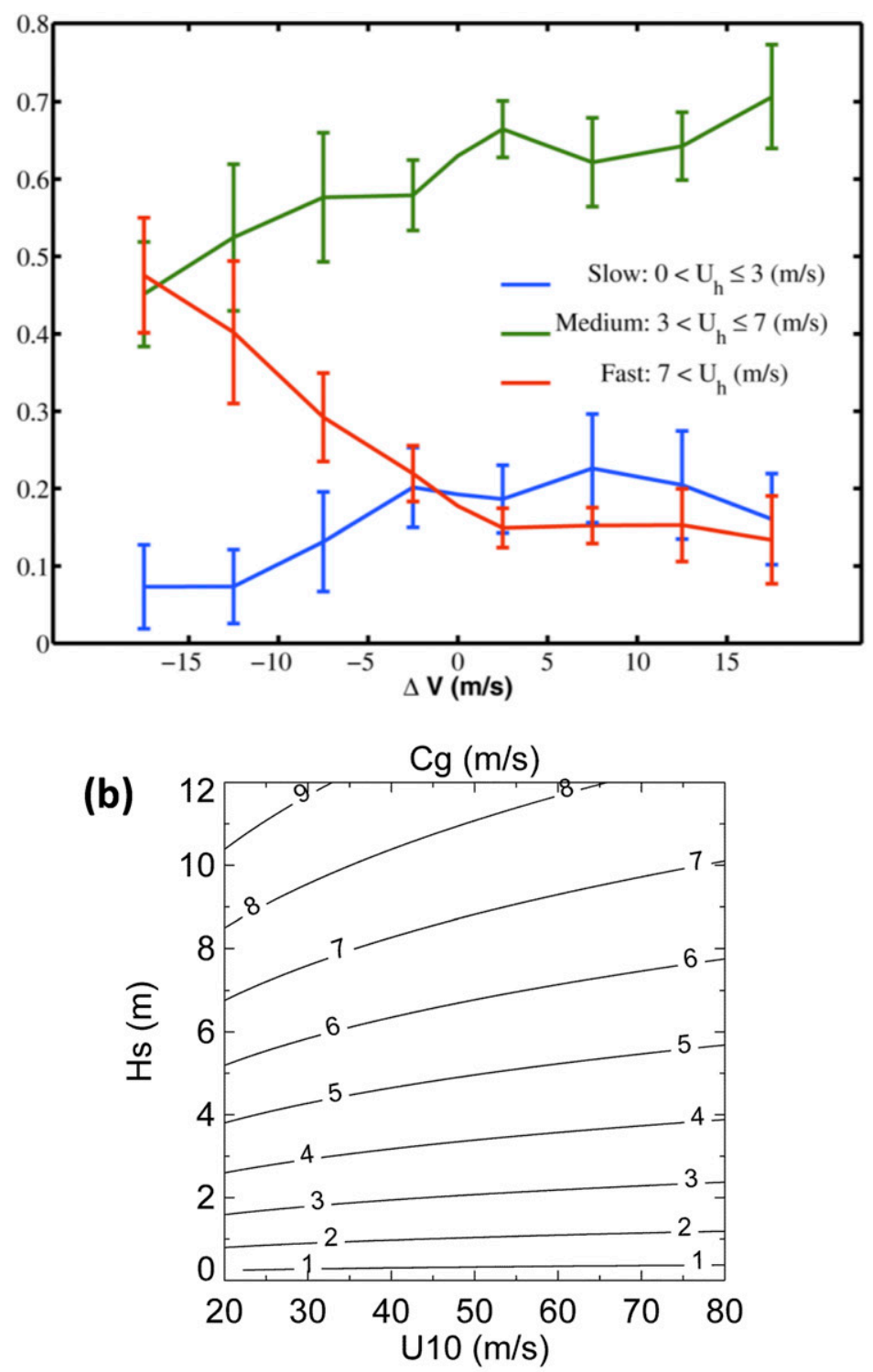

(c)

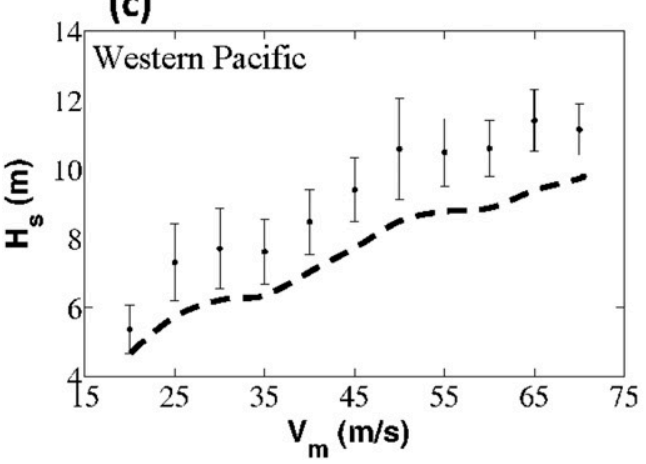

(d)



FIG. 6. (a) Percentages of the three indicated storms' translating speed groups in western Pacific and North Atlantic plotted as a function of intensity-change $\Delta V$ in $5 \mathrm{~m} \mathrm{~s}^{-1}$ bins. Vertical lines show \pm 1 sd. (b) Group speeds from Zhang and Oey (2018): $C_{g}=4.6459 H_{s}^{0.5848} / U_{10}^{0.1696}$, multiplied by $\cos \left(35^{\circ}\right)$ (see text), plotted as a function of the observed significant wave height $H_{s}$ and reference wind speed $U_{10}$. (c),(d) The mean 90th percentile $H_{s}$ (filled circles with \pm 1 sd lines) observed in the right-front quadrant of tropical cyclones in the (c) western Pacific and (d) North Atlantic, plotted as a function of the maximum tropical cyclone wind $V_{m}$ in $5 \mathrm{~m} \mathrm{~s}^{-1}$ bins. Dashed lines show the mean 50th percentile $H_{s}$ (without the $\pm 1 \mathrm{sd}$ lines for clarity). 
considered, $C_{g}$ varies between 3 and $8 \mathrm{~ms}^{-1}$, approximately in the range of $U_{h}$ of the Goldilocks tropical cyclones. Finally, Figs. $6 \mathrm{c}$ and $6 \mathrm{~d}$ show that as $V_{m}$ increases, the $H_{s}$ also increases, nearly linearly up to about $45 \mathrm{~m} \mathrm{~s}^{-1}$, and then $H_{s}$ plateaus or saturates at $\sim 10-12 \mathrm{~m}$ for stronger wind speeds $50 \mathrm{~m} \mathrm{~s}^{-1}$ and larger. ${ }^{3}$ The best fit of the 90th percentile curve is $V_{m} \sim H_{s}^{2}$ (i.e., $k$ is likely to be $>1 / 2$ ). Instead of $V_{m}$, estimated $U_{10}$ also gives a similar variation $U_{10} \sim H_{s}^{2}$ (Zhang and Oey 2018). The highwave saturation guarantees that, under the Goldilocks condition, more young waves appear in the right-front quadrant where $\omega_{n}$, hence $\alpha_{M}$, becomes large. The "spilling" of high $\alpha_{M}$ into the right-front quadrant (Fig. 5, near the 2 o'clock position) allows a high coincidence of the $\alpha_{M}$ with the MFC, further intensifying the cyclone (Fig. 5, back to the 7 o'clock position). We suggest that this positive reinforcing loop between the tropical cyclone wind and young waves in the right-front quadrant favors rapid intensification.

Indicated in Fig. 5 is also the potential intensity-enhancing effect of spray as large amounts are spewed into "the nearsurface droplet evaporation layer" (Andreas 2011) at high wind speeds (Andreas and Emanuel 2001; Donelan et al. 2004; Holthuijsen et al. 2012; Soloviev et al. 2014). In addition to potentially reducing the surface drag (Soloviev et al. 2014), discussed below, the spray induces spraymediated enthalpy flux (in addition to the customary interfacial flux), which at high winds, $U_{10} \sim 35 \mathrm{~ms}^{-1}$ and higher, scales like $U_{10}^{\gamma}$, with $\gamma$ between $\sim 2$ and 4 (Andreas 2010; Richter and Stern 2014). Despite this robust, increasing trend of the enthalpy flux with $U_{10}$ from field measurements, the corresponding trend in the graph of the enthalpy flux coefficient $C_{k}$ versus $U_{10}$ is statistically insignificant because of large scatter in the field data (Richter and Stern 2014), in part due to the very difficult conditions under which field measurements were often taken (Jarosz et al. 2007; Andreas 2010; Holthuijsen et al. 2012). A recent laboratory experiment by Komori et al. (2018) provides some evidence of enhanced enthalpy flux at high winds. They showed that $C_{k}$ is approximately constant for wind speeds below $\sim 35 \mathrm{~ms} \mathrm{~s}^{-1}$, but increases linearly with the wind speed for stronger wind. The result appears to be consistent with Andreas's (2011) analysis, which shows a significant increase in $C_{k}$ with $U_{10}$ once the spray-mediated

\footnotetext{
${ }^{3}$ Donelan et al. (2004) vividly described the process: “...a breaking wave, with abrupt change in slope., causes the (air) flow to separate from the surface and reattach near the crest of the preceding wave... The outer flow, unable to follow the wave surface, does not 'see' the troughs of the waves and skips from breaking crest to breaking crest..." (p. 4). The upshot is a reduction in the Jeffreys's (1925) sheltering effect and the form drag (Donelan 2018), and the large waves become saturated.
}

fluxes begin to dominate over the interfacial fluxes. It is interesting that, since $\alpha_{M} \sim U_{10} /\left(g H_{s}\right)^{1 / 2}$, the exchange coefficient we have used (i.e. Hwang and Walsh 2016) also scales linearly with $U_{10}$ when waves become saturated. Komori et al.'s result is consistent with Andreas (2010) and Richter and Stern (2014), but is in contrast to what has customarily been assumed based on data at lower wind speeds (e.g., Drennan et al. 2007; Jeong et al. 2012), that the enthalpy coefficient remains constant at high winds (e.g., Soloviev et al. 2014, 2017; Donelan 2018). However, conditions in laboratory experiments are not the same as those in the field (Richter and Stern 2014). How spray may affect tropical cyclone intensity is complex, and remains a topic of intense debate and research (e.g., Wang et al. 2001; Gall et al. 2008; Garg et al. 2018).

The proposed wind-wave reinforcing mechanism of Fig. 5 appears similar to the WISHE evaporation-wind feedback mechanism, previewed in the introduction. However, WISHE relies on the feedback between the wind and surface enthalpy flux $\left(Q_{k}\right)$ that is a function of the wind speed $\left|\mathbf{U}_{10}\right|$ :

$$
Q_{k}=\rho_{a} C_{k}\left(k_{s}-k_{a}\right)\left|\mathbf{U}_{10}\right|
$$

where $\rho_{a}$ is the air density; $C_{k}$ is the dimensionless exchange coefficient for enthalpy; and $k_{s}$ and $k_{a}$ are the specific enthalpies of the air at saturation at the ocean surface and ambient boundary layer air, respectively. On the other hand, the wind-wave reinforcing mechanism depends on the increased wave-induced air-sea exchange coefficient $\alpha_{M}$ and its coincidence with the MFC. Unlike WISHE, the wind-wave reinforcing mechanism is nonaxisymmetric. While one might anticipate a functional relation between $C_{k}$ and $\alpha_{M}\left(\sim\left|\mathbf{U}_{10}\right|\right.$ as waves saturate; in which case WISHE can augment intensification; Zhang and Emanuel 2016), the presence of young waves in the windwave reinforcing mechanism injects new processes (e.g., spray) as outlined above; thus, further research is needed.

In Emanuel's (1986, 1999) heat engine model, the maximum steady intensity a storm can achieve is

$$
V_{\text {pot }}^{2}=\left(C_{k} / C_{D}\right)\left(T_{s}-T_{o}\right)\left(k_{s}-k_{a}\right) / T_{o},
$$

where $V_{\text {pot }}$ is the maximum wind speed, and $T_{s}$ and $T_{o}$ are the absolute temperatures of the sea surface and storm top. Soloviev et al. $(2014,2017)$ and Donelan (2018) proposed that $C_{D}$ peaks around a wind speed of $\sim 30-40 \mathrm{~m} \mathrm{~s}^{-1}$, but reaches some minimum around $\sim 50-$ $60 \mathrm{~m} \mathrm{~s}^{-1}$ before increasing again. Thus, if $C_{k}$ is assumed constant, then $V_{\text {pot }}$ may amplify, sufficiently rapidly to explain the observed bimodal distribution of the lifetime maximum intensity of tropical cyclones (Kossin et al. 2013). In view of the above discussion that $C_{k}$ is not constant at high winds, but varies like $\sim U_{10}^{k}$ with 

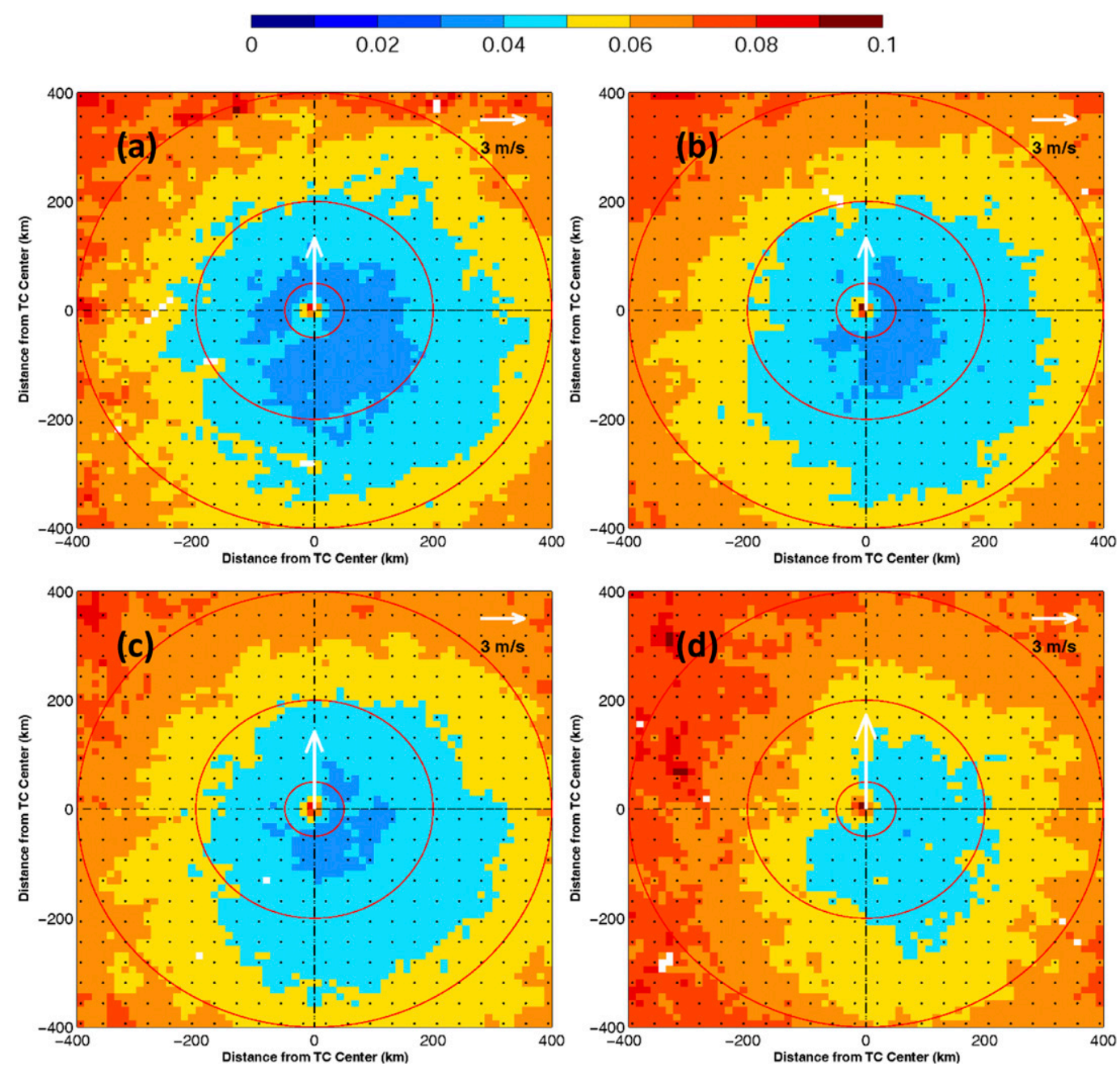

FIG. 7. The Stokes speed divided by wind speed $\left|\mathbf{u}_{s}\right| / U_{10}$ for the four different tropical cyclone intensity change groups: (a) RI, (b) SMI, (c) N, and (d) W. Vector at center shows tropical cyclone heading and its length is the averaged translation speed with the scale at top right. The plots show that the $\left|\mathbf{u}_{s}\right| / U_{10}$ hence also the ocean mixing and therefore cooling caused by the Stokes current is least in the right-back quadrant where waves are youngest (Figs. S1a-d). This agrees with numerical experiments showing also the least amount of sea surface temperature cooling in the right-back quadrant (Fan et al. 2009). We see then that the RI group in (a) with the youngest waves among the four groups has the weakest Stokes current relative to the wind, and therefore also the least fractional cooling relative to the wind. In other words, for the same wind speed, the RI tropical cyclones are more immune to intensity-inhibiting surface cooling because of the widespread presence of young waves in its core.

the power $k$ most likely $\geq 1$, the lone effect of $C_{D}$ in explaining rapid intensification is unclear. In Soloviev et al.'s theory, the large spray droplets (spume) that contribute to smoothing the sea surface and drag reduction are also those that play a central role in the spray-mediated enthalpy fluxes that potentially can enhance $C_{k}$ (Andreas and Emanuel 2001; Andreas 2011). It is likely that both a decrease in $C_{D}$ and an increase in $C_{k}$ at high wind speeds contribute to TC intensification process. Hence, more research is needed.

Instead of examining the lifetime maximum intensity, our study focuses on actual intensity changes $(\Delta V)$, and our finding suggests a close coupling between waves and the MFC for the rapid intensification of the tropical cyclone. Young waves play a central role in our results. Waves drive ocean Stokes current, $\mathbf{u}_{s}$ (Stokes 1880), which contributes to mixing that cools the sea surface (Tamura et al. 2012). A formula for the Stokes speed at the sea surface can be derived from the second form of Eq. (2) relating the significant wave height with inverse wave age; thus, $\left|\mathbf{u}_{s}\right| / U_{10}=2.94 \times 10^{-3} \omega_{n}^{-0.42}$. For a given cyclone wind, the Stokes speed is least in the right-back quadrant where waves are youngest (Fig. 7). This prediction is supported by full-physics 
numerical experiments that showed also that the least amount of sea surface temperature cooling was in the right-back quadrant, caused by wave-induced mixing (Fan et al. 2009). For young waves, since a fraction of wind energy is spent in growing them, there is less available for mixing and cooling the sea surface. Compared to non-rapidly intensifying storms, a rapidly intensifying storm with abundant young waves in its core has therefore relatively weaker Stokes current (i.e., for the same wind speed) and is therefore more immune to intensity-inhibiting surface cooling. Our results suggest that a new surface layer parameterization including young waves may be developed to improve tropical cyclone intensity forecast.

Acknowledgments. The authors are grateful for correspondence with Paul Hwang, whose work (Hwang and Walsh's 2016 in particular) has inspired this study. We thank Prof. Soloviev and the anonymous reviewer 2 for providing useful comments, which improved the manuscript. Data used here are publically available from AVISO (http://www.aviso.altimetry.fr/en/my-aviso.html), GHRSST (https://www.ghrsst.org/), NCEP (https://rda. ucar.edu/datasets/ds083.2/), and NDBC (http://www. ndbc.noaa.gov/). This research was supported by the Taiwan Ministry of Science and Technology Grants 107-2111-M-008-035 and 107-2611-M-008-003, awarded to the National Central University. Author contributions: LO conceived the idea, designed the study, interpreted the data, and wrote the paper. LZ analyzed the data, conducted the WRF simulations, and plotted the figures, with some contributions from LO. Both authors derived the equations and discussed the research.

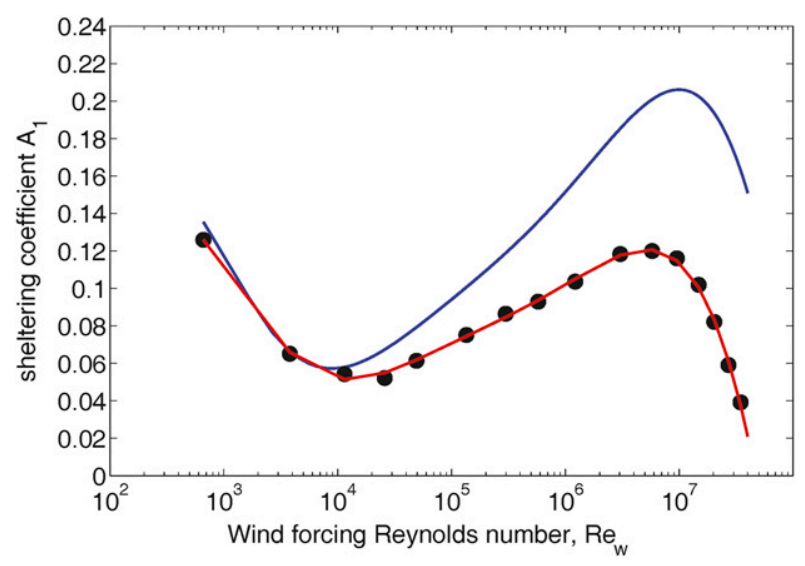

FIG. A1. The Donelan's (2018) sheltering coefficient $A_{1}$ vs Reynolds number $\mathrm{Re}_{w}$ from ocean measurements. The black dots are Donelan's data points from his Fig. 5. The red line is the fitted septic formula: $A_{1}=8.2987 \times 10^{-7} x^{7}-7.4548 \times$ $10^{-5} x^{6}+2.7999 \times 10^{-3} x^{5}-5.7021 \times 10^{-2} x^{4}+6.7957 \times 10^{-1} x^{3}$ $-4.7287 x^{2}+17.722 x-27.392$, for $662 \leq \mathrm{Re}_{w} \leq 4 \times 10^{7}$, where $x=\ln \left(\operatorname{Re}_{w}\right)$. The dark blue line is based on the late Professor Donelan's fitted formula, Eq. (13) of Donelan (2018), which appears to have typos.

\section{APPENDIX}

\section{The Sheltering Coefficient $A_{1}$ and the Reynolds Number-Dependent, Modified $\alpha_{M}: \alpha_{\mathrm{MM}}$}

Here we incorporate the Donelan's (2018) Reynolds number-dependent sheltering coefficient $A_{1}$ (see Donelan et al. 2012) into the Hwang and Walsh's (2016) $\alpha_{M}$ to obtain the Reynolds number-dependent $\alpha_{\mathrm{MM}}$. We first fitted Donelan's (2018, his Fig. 5) data to obtain the following for the sheltering coefficient $A_{1}$ :

$$
\begin{array}{rlrl}
A_{1}= & 0.1259, & 0<\mathrm{Re}_{w}<662, \\
A_{1}= & 8.2987 \times 10^{-7} x^{7}-7.4548 \times 10^{-5} x^{6}+2.7999 \times 10^{-3} x^{5}-5.7021 \times 10^{-2} x^{4}+ \\
& 6.7957 \times 10^{-1} x^{3}-4.7287 x^{2}+17.722 x-27.392 ; & & 662 \leq \mathrm{Re}_{w} \leq 4 \times 10^{7}, \\
A_{1}= & 0.02086, & & 4 \times 10^{7}<\mathrm{Re}_{w},
\end{array}
$$

where $x=\ln \left(\operatorname{Re}_{w}\right)$. The septic formula is plotted in Fig. A1, which corrects the late Professor Donelan's (2018) fitted formula (dark blue; which appears to contain typos). Here, $\mathrm{Re}_{w}$ is the Reynolds number:

$$
\operatorname{Re}_{w}=\left(U_{20}-C_{p}\right) \times H_{s} / \nu_{a},
$$

where $U_{20}=20-\mathrm{m}$ wind speed, $\nu_{a}=1.48 \times 10^{-5} \mathrm{~m}^{2} \mathrm{~s}^{-1}$ is the kinematic viscosity of air, $H_{s}$ is the significant wave height from satellite data, and $C_{p}$ is the wave phase speed estimated from $U_{10}, H_{s}$, and the wave-growth function (see the main text; Zhang and Oey 2018). For simplicity the 20-m height for the wind speed is used in place of the wind at half-peak wavelength height $\left(U_{\lambda / 2}\right)$ used in Donelan (2018), following the recommendation of Donelan et al. (2012) [the $U_{\lambda / 2}$ may be calculated also more precisely using the dispersion relation; Mellor et al. (2008)]. The logarithmic law is used to estimate $U_{20}$ from $U_{10}$ :

$$
U_{20}=\left(1+1.733 C_{D}^{1 / 2}\right) \times U_{10},
$$

where the drag coefficient 


$$
\begin{array}{ll}
C_{D}=1.2 \times 10^{-3} & U_{10} \leq 4.5 \mathrm{~m} \mathrm{~s}^{-1}, \\
C_{D}=10^{-5} \times\left(-0.16 U_{10}^{2}+9.67 U_{10}+80.58\right), & 4.5<U_{10} \leq 61.5 \mathrm{~m} \mathrm{~s}^{-1}, \\
C_{D}=7 \times 10^{-4} & 61.5 \mathrm{~m} \mathrm{~s}^{-1}<U_{10} .
\end{array}
$$

The first form of the $C_{D}$ formula is shown for completeness only to fit the Large and Pond's (1981) value, as the $U_{10}$ in our analysis all exceed $4.5 \mathrm{~m} \mathrm{~s}^{-1}$. The second form is from Hwang and Walsh (2016); it fits for example, Powell et al. (2003) and others' data, peaking at around $2.3 \times 10^{-3}$ for $U_{10} \approx 30 \mathrm{~m} \mathrm{~s}^{-1}$ and decreasing for higher wind speeds. The last form uses Holthuijsen et al.'s (2012) value for the minimum $C_{D}$. The sheltering coefficient is thus completely determined from the given $H_{s}$ from satellite data.

The Reynolds number-dependent $\alpha_{\mathrm{MM}}$ is calculated by noting that for a sinusoidal motion the wave energy is equal to wave phase speed times the wave momentum (Hwang and Walsh 2016); the $\alpha_{\mathrm{MM}}$ can then be related to the wind energy input to the waves, and is directly proportional to $A_{1}$ (c.f. Donelan et al. 2012). After some algebra to reconcile the two different formulations (Donelan et al. 2012; Hwang and Walsh 2016) we get

$$
\alpha_{\mathrm{MM}}\left(\mathrm{Re}_{w}\right)=9.36 \times A_{1}\left(\mathrm{Re}_{w}\right) \times \alpha_{M},
$$

where "9.36" is the proportionality factor, and the Reynolds number-independent $\alpha_{M}$ was calculated as described in the text. It is interesting that the proportionality factor 9.36 makes $\alpha_{\mathrm{MM}}=\alpha_{M}$ at $\mathrm{Re}_{w}=10^{6}$ (roughly corresponding to $U_{10} \approx 30 \mathrm{~m} \mathrm{~s}^{-1}$ ), prior to the $A_{1}$ maximum near $\mathrm{Re}_{w}=6 \times 10^{6}$ and the anticipated drop at higher wind speeds.

\section{REFERENCES}

Andreas, E. L., 2010: Spray-mediated enthalpy flux to the atmosphere and salt flux to the ocean in high winds. J. Phys. Oceanogr., 40, 608-619, https://doi.org/10.1175/2009JPO4232.1.

- 2011: Fallacies of the enthalpy transfer coefficient over the ocean in high winds. J. Atmos. Sci., 68, 1435-1445, https://doi.org/ 10.1175/2011JAS3714.1.

— , and K. A. Emanuel, 2001: Effects of sea spray on tropical cyclone intensity. J. Atmos. Sci., 58, 3741-3751, https://doi.org/ 10.1175/1520-0469(2001)058<3741:EOSSOT>2.0.CO;2.

Atlas, R., R. N. Hoffman, J. Ardizzone, S. M. Leidner, J. C. Jusem, D. K. Smith, and D. Gombos, 2011: A cross-calibrated, multiplatform ocean surface wind velocity product for meteorological and oceanographic applications. Bull. Amer. Meteor. Soc., 92, 157-174, https://doi.org/10.1175/2010BAMS2946.1.

Barenblatt, G. I., A. J. Chorin, and V. M. Prostokishin, 2005: A note concerning the Lighthill 'sandwich model' of tropical cyclones. Proc. Natl. Acad. Sci. USA, 102, 11148-11150, https://doi.org/10.1073/pnas.0505209102.

Batchelor, G. K., 1967: An Introduction to Fluid Dynamics. Cambridge University Press, 615 pp.
Black, P. G., and Coauthors, 2007: Air-sea exchange in hurricanes: Synthesis of observations from the Coupled Boundary Layer Air-Sea Transfer experiment. Bull. Amer. Meteor. Soc., 88, 357-374, https://doi.org/10.1175/BAMS-88-3-357.

Bowyer, P. J., and A. W. MacAfee, 2005: The theory of trapped-fetch waves with tropical cyclones-An operational perspective. Wea. Forecasting, 20, 229-244, https://doi.org/10.1175/WAF849.1.

Chu, P. S., 2002: Large-scale circulation features associated with decadal variations of tropical cyclone activity over the central North Pacific. J. Climate, 15, 2678-2689, https://doi.org/10.1175/ 1520-0442(2002)015<2678:LSCFAW >2.0.CO;2.

Donelan, M., 2018: On the decrease of the oceanic drag coefficient in high winds. J. Geophys. Res. Oceans, 123, 1485-1501, https:// doi.org/10.1002/2017JC013394.

_, M. Skafel, H. Graber, P. Liu, D. Schwab, and S. Venkates, 1992: On the growth of wind-generated waves. Atmos.-Ocean, 30, 457-478, https://doi.org/10.1080/07055900.1992.9649449.

, B. Haus, N. Reul, W. Plant, M. Stiassnie, H. Graber, O. Brown, and E. Saltzman, 2004: On the limiting aerodynamic roughness of the ocean in very strong winds. Geophys. Res. Lett., 31, L18306, https://doi.org/10.1029/ 2004GL019460.

_- M. Curcic, S. S. Chen, and A. K. Magnusson, 2012: Modeling waves and wind stress. J. Geophys. Res., 117, C00J23, https:// doi.org/10.1029/2011JC007787.

Drennan, W. M., J. Zhang, J. R. French, C. McCormick, and P. G. Black, 2007: Turbulent fluxes in the hurricane boundary layer. Part II: Latent heat flux. J. Atmos. Sci., 64, 1103-1115, https:// doi.org/10.1175/JAS3889.1.

Dunion, J. P., and C. S. Velden, 2004: The impact of the Saharan air layer on Atlantic tropical cyclone activity. Bull. Amer. Meteor. Soc., 85, 353-366, https://doi.org/10.1175/BAMS-85-3-353.

Emanuel, K. A., 1986: An air-sea interaction theory for tropical cyclones. Part I: Steady-state maintenance. J. Atmos. Sci., $\mathbf{4 3}$, 585-605, https://doi.org/10.1175/1520-0469(1986)043<0585: AASITF $>2.0 . \mathrm{CO} ; 2$.

, 1991: The theory of hurricanes. Annu. Rev. Fluid Mech., 23, 179-196, https://doi.org/10.1146/annurev.fl.23.010191.001143. , 1999: Thermodynamic control of hurricane intensity. Nature, 401, 665-669, https://doi.org/10.1038/44326.

Evans, J. L., and Coauthors, 2010: Environmental impacts on tropical cyclone structure and intensity change. Seventh Int. Workshop on Tropical Cyclones, La Reunion, France, WMO/ CAS/WWW, 20 pp.

Fan, Y., I. Ginis, and T. Hara, 2009: The effect of wind-wavecurrent interaction on air-sea momentum flux and ocean response in tropical cyclones. J. Phys. Oceanogr., 39, 1019-1034, https://doi.org/10.1175/2008JPO4066.1.

Fiorino, M., and R. L. Elsberry, 1989: Some aspects of vortex structure related to tropical cyclone motion. J. Atmos. Sci., 46, 975-990, https://doi.org/10.1175/1520-0469(1989)046<0975: SAOVSR $>2.0 . \mathrm{CO} ; 2$.

Frank, W. M., and E. A. Ritchie, 2001: Effects of vertical wind shear on the intensity and structure of numerically simulated hurricanes. Mon. Wea. Rev., 129, 2249-2269, https://doi.org/ 10.1175/1520-0493(2001)129<2249:EOVWSO>2.0.CO;2. 
Gall, J. S., W. M. Frank, and Y. Kwon, 2008: Effect of sea spray on tropical cyclones simulated under idealized conditions. Mon. Wea. Rev., 136, 1686-1705, https://doi.org/10.1175/2007MWR2183.1.

Gall, R., J. Franklin, F. Marks, E. N. Rappaport, and F. Toepfer, 2013: The Hurricane Forecast Improvement Project. Bull. Amer. Meteor. Soc., 94, 329-343, https://doi.org/10.1175/ BAMS-D-12-00071.1.

Garg, N., E. Y. K. Ng, and S. Narasimalu, 2018: The effect of sea spray and atmosphere-wave coupling on air-sea exchange during a tropical cyclone. Atmos. Chem. Phys., 18, 6001-6021, https://doi.org/10.5194/acp-18-6001-2018.

Goni, G., S. Kamholz, S. Garzoli, and D. Olson, 1996: Dynamics of the Brazil-Malvinas Confluence based on inverted echo sounders and altimetry. J. Geophys. Res., 101, 16273-16289, https://doi.org/10.1029/96JC01146.

Hendricks, E. A., M. T. Montgomery, and C. A. Davis, 2004: The role of vortical hot towers in the formation of Tropical Cyclone Diana (1984). J. Atmos. Sci., 61, 1209-1232, https://doi.org/ 10.1175/1520-0469(2004)061<1209:TROVHT>2.0.CO;2.

— - M. S. Peng, B. Fu, and T. Li, 2010: Quantifying environmental control on tropical cyclone intensity change. Mon. Wea. Rev., 138, 3243-3271, https://doi.org/10.1175/2010MWR3185.1.

Holland, G. J., J. I. Belanger, and A. Fritz, 2010: A revised model for radial profiles of hurricane winds. Mon. Wea. Rev., 138, 4393-4401, https://doi.org/10.1175/2010MWR3317.1.

Holthuijsen, L. H., M. D. Powell, and J. D. Pietrzak, 2012: Wind and waves in extreme hurricanes. J. Geophys. Res., 117, C09003, https://doi.org/10.1029/2012JC007983.

Houze, R. A., S. S. Chen, B. F. Smull, W. C. Lee, and M. M. Bell, 2007: Hurricane intensity and eyewall replacement. Science, 315, 1235-1239, https://doi.org/10.1126/science.1135650.

Hwang, P. A., and D. W. Wang, 2004: Field measurements of duration limited growth of wind-generated ocean surface waves at young stage of development. J. Phys. Oceanogr., 34, 2316-2326, https:// doi.org/10.1175/1520-0485(2004)034<2316:FMODGO>2.0.CO;2.

_ , and E. J. Walsh, 2016: Azimuthal and radial variation of wind-generated surface waves inside tropical cyclones. J. Phys. Oceanogr., 46, 2605-2621, https://doi.org/10.1175/JPO-D-16-0051.1.

Jakobsen, F., and H. Madsen, 2004: Comparison and further development of parametric tropical cyclone models for storm surge modelling. J. Wind Eng. Ind. Aerodyn., 92, 375-391, https://doi.org/10.1016/j.jweia.2004.01.003.

Jarosz, E., D. A. Mitchell, D. W. Wang, and W. J. Teague, 2007: Bottom-up determination of air-sea momentum exchange under a major tropical cyclone. Science, 315, 1707-1709, https://doi.org/10.1126/science.1136466.

Jeffreys, H., 1925: On the formation of waves by wind. Proc. Roy. Soc. London, 107A, 189-206, https://doi.org/10.1098/rspa.1925.0015.

Jeong, D., B. K. Haus, and M. A. Donelan, 2012: Enthalpy transfer across the air-water interface in high winds including spray. J. Atmos. Sci., 69, 2733-2748, https://doi.org/10.1175/JAS-D11-0260.1.

Kaplan, J., and M. DeMaria, 2003: Large-scale characteristics of rapidly intensifying tropical cyclones in the North Atlantic basin. Wea. Forecasting, 18, 1093-1108, https://doi.org/ 10.1175/1520-0434(2003)018<1093:LCORIT >2.0.CO;2.

Knaff, J. A., C. R. Sampson, M. DeMaria, T. P. Marchok, J. M. Gross, and C. J. McAdie, 2007: Statistical tropical cyclone wind radii prediction using climatology and persistence. Wea. Forecasting, 22, 781-791, https://doi.org/10.1175/WAF1026.1.

Knapp, K. R., M. C. Kruk, D. H. Levinson, H. J. Diamond, and C. J. Neumann, 2010: The International Best Track Archive for Climate Stewardship (IBTrACS) unifying tropical cyclone data. Bull. Amer. Meteor. Soc., 91, 363-376, https://doi.org/ 10.1175/2009BAMS2755.1.

Komori, S., K. Iwano, N. Takagaki, R. Onishi, R. Kurose, K. Takahashi, and N. Suzuki, 2018: Laboratory measurements of heat transfer and drag coefficients at extremely high wind speeds. J. Phys. Oceanogr., 48, 959-974, https://doi.org/ 10.1175/JPO-D-17-0243.1.

Kossin, J. P., T. L. Olander, and K. R. Knapp, 2013: Trend analysis with a new global record of tropical cyclone intensity. J. Climate, 26, 9960-9976, https://doi.org/10.1175/JCLI-D-13-00262.1.

Kowch, R., and K. Emanuel, 2015: Are special processes at work in the rapid intensification of tropical cyclones? Mon. Wea. Rev., 143, 878-882, https://doi.org/10.1175/MWR-D-14-00360.1.

Large, W. G., and S. Pond, 1981: Open ocean flux measurements in moderate to strong winds. J. Phys. Oceanogr., 11, 324-336, https:// doi.org/10.1175/1520-0485(1981)011<0324:OOMFMI>2.0.CO;2.

Lee, C.-Y., M. K. Tippett, A. H. Sobel, and S. J. Camargo, 2016: Rapid intensification and the bimodal distribution of tropical cyclone intensity. Nat. Commun., 7, 10625, https://doi.org/10.1038/ ncomms10625.

Lighthill, J., 1999: Ocean spray and the thermodynamics of tropical cyclones. J. Eng. Math., 35, 11-42, https://doi.org/10.1023/ A:1004383430896.

Lin, I., C.-C. Wu, I.-F. Pun, and D.-S. Ko, 2008: Upper-ocean thermal structure and the western North Pacific category 5 typhoons. Part I: Ocean features and the category 5 typhoons' intensification. Mon. Wea. Rev., 136, 3288-3306, https://doi.org/ 10.1175/2008MWR2277.1.

Longuet-Higgins, M. S., 1952: On the statistical distribution of the heights of sea waves. J. Mar. Res., 11, 245-266.

Mellor, G. L., M. Donelan, and L. Y. Oey, 2008: A surface wave model for coupling with numerical ocean circulation models. J. Atmos. Oceanic Technol., 25, 1785-1807, https://doi.org/ 10.1175/2008JTECHO573.1.

Merrill, R. T., 1988: Characteristics of the upper-tropospheric environmental flow around hurricanes. J. Atmos. Sci., 45, 1665-1677, https:// doi.org/10.1175/1520-0469(1988)045<1665:COTUTE > 2.0.CO;2.

Montgomery, M. T., and R. K. Smith, 2014: Paradigms for tropical cyclone intensification. Aust. Meteor. Ocean J., 64, 37-66, https://doi.org/10.22499/2.6401.005.

— S. V. Nguyen, R. K. Smith, and J. Persing, 2009: Do tropical cyclones intensify by WISHE? Quart. J. Roy. Meteor. Soc., 135, 1697-1714, https://doi.org/10.1002/qj.459.

_ J. Jersing, and R. K. Smith, 2015: Putting to rest WISHE-ful misconceptions for tropical cyclone intensification. J. Adv. Model. Earth Syst., 7, 92-109, https://doi.org/10.1002/2014MS000362.

Moon, I.-J., I. Ginis, and T. Hara, 2003: Numerical simulation of sea surface directional wave spectra under hurricane wind forcing. J. Phys. Oceanogr., 33, 1680-1706, https://doi.org/10.1175/2410.1.

Oey, L.-Y., T. Ezer, D.-P. Wang, X.-Q. Yin, and S.-J. Fan, 2007: Hurricane-induced motions and interaction with ocean currents. Cont. Shelf Res., 27, 1249-1263, https://doi.org/10.1016/ j.csr.2007.01.008.

Peng, M. S., B. Jeng, and R. T. Williams, 1999: A numerical study on tropical cyclone intensification. Part I: Beta effect and mean flow effect. J. Atmos. Sci., 56, 1404-1423, https://doi.org/ 10.1175/1520-0469(1999)056<1404:ANSOTC >2.0.CO;2.

Powell, M. D., P. J. Vickery, and T. A. Reinhold, 2003: Reduced drag coefficient for high wind speeds in tropical cyclones. Nature, 422, 279-283, https://doi.org/10.1038/nature01481.

Press, W. H., S. A. Teukolsky, W. T. Vetterling, and B. P. Flannery, 1992: Numerical Recipes: The Art of Scientific Computing. Cambridge University Press, 933 pp. 
Rappaport, E. N., and Coauthors, 2009: Advances and challenges at the National Hurricane Center. Wea. Forecasting, 24, 395419, https://doi.org/10.1175/2008WAF2222128.1.

Rappin, E. D., M. C. Morgan, and G. J. Tripoli, 2011: The impact of outflow environment on tropical cyclone intensification and structure. J. Atmos. Sci., 68, 177-194, https://doi.org/10.1175/ 2009JAS2970.1.

Richter, D. H., and D. P. Stern, 2014: Evidence of spray-mediated air-sea enthalpy flux within tropical cyclones. Geophys. Res. Lett., 41, 2997-3003, https://doi.org/10.1002/2014GL059746.

Riehl, H., 1963: Some relations between wind and thermal structure of steady state hurricanes. J. Atmos. Sci., 20, 276-287, https://doi.org/ 10.1175/1520-0469(1963)020<0276:SRBWAT $>2.0 . C O ; 2$.

Rios-Berrios, R., T. Vukicevic, and B. Tang, 2014: Adopting model uncertainties for tropical cyclone intensity prediction. Mon. Wea. Rev., 142, 72-78, https://doi.org/10.1175/MWR-D-1300186.1.

Rogers, R., and Coauthors, 2006: The intensity forecasting experiment: A NOAA multiyear field program for improving tropical cyclone intensity forecasts. Bull. Amer. Meteor. Soc., 87, 1523-1537, https://doi.org/10.1175/BAMS-87-11-1523.

Schade, L. R., and K. A. Emanuel, 1999: The ocean's effect on the intensity of tropical cyclones: Results from a simple coupled atmosphere-ocean model. J. Atmos. Sci., 56, 642-651, https:// doi.org/10.1175/1520-0469(1999)056<0642:TOSEOT>2.0.CO;2.

Shay, L. K., G. J. Goni, and P. G. Black, 2000: Effects of a warm oceanic feature on Hurricane Opal. Mon. Wea. Rev., 128, 1366-1383, https://doi.org/10.1175/1520-0493(2000)128<1366: EOAWOF $>2.0 . \mathrm{CO} ; 2$.

Soloviev, A. V., R. Lukes, M. A. Donelan, B. K. Haus, and I. Ginis, 2014: The air-sea interface and surface stress under tropical cyclones. Sci. Rep., 4, 5306, https://doi.org/10.1038/srep05306.

, R. Lukas, M. A. Donelan, B. K. Haus, and I. Ginis, 2017: Is the state of the air-sea interface a factor in rapid intensification and rapid decline of tropical cyclones? J. Geophys. Res. Oceans, 122, 10 174-10183, https://doi.org/10.1002/2017JC013435.

Stephens, C., J. I. Antonov, T. P. Boyer, M. E. Conkright, R. A. Locarnini, T. D. O'Brien, H. E. Garcia, and S. Levitus, 2001: Temperature. Vol. 1, World Ocean Atlas 2001, NOAA Atlas NESDIS 49, 176 pp.

Stokes, G. G., 1880: On the theory of oscillatory waves. Mathematical and Physical Papers, Vol. I. Cambridge University Press, 197229, https://doi.org/10.1017/CBO9780511702242.013.

Sun, J., and L.-Y. Oey, 2015: The influence of the ocean on Typhoon Nuri (2008). Mon. Wea. Rev., 143, 4493-4513, https:// doi.org/10.1175/MWR-D-15-0029.1.
,-- , F.-H. Xu, and Y.-C. Lin, 2017: Sea level rise, surface warming, and the weakened buffering ability of South China Sea to strong typhoons in recent decades. Sci. Rep., 7, 7418, https://doi.org/10.1038/s41598-017-07572-3.

Takagaki, N., S. Komori, N. Suzuki, K. Iwano, T. Kuramoto, S. Shimada, R. Kurose, and K. Takahashi, 2012: Strong correlation between the drag coefficient and the shape of the wind sea spectrum over a broad range of wind speeds. Geophys. Res. Lett., 39, L23604, https://doi.org/10.1029/2012GL053988.

$-, \ldots,-, \ldots$, and R. Kurose, 2016: Mechanism of drag coefficient saturation at strong wind speeds. Geophys. Res. Lett., 43, 9829-9835, https://doi.org/10.1002/2016GL070666.

Tamura, H., Y. Miyazawa, and L.-Y. Oey, 2012: The Stokes drift and wave induced-mass flux in the North Pacific. J. Geophys. Res., 117, C08021, https://doi.org/10.1029/2012JC008113.

Toba, Y., 1972: Local balance in the air-sea boundary processes I. On the growth process of wind waves. J. Oceanogr. Soc. Japan, 28, 109-120, https://doi.org/10.1007/BF02109772.

Wang, Y., and C.-C. Wu, 2004: Current understanding of tropical cyclone structure and intensity changes-A review. Meteor. Atmos. Phys., 87, 257-278, https://doi.org/10.1007/s00703-0030055-6.

_ J. D. Kepert, and G. J. Holland, 2001: The effect of sea spray evaporation on tropical cyclone boundary layer structure and intensity. Mon. Wea. Rev., 129, 2481-2500, https://doi.org/ 10.1175/1520-0493(2001)129<2481:TEOSSE $>2.0$. CO;2.

Wu, L. G., and S. A. Braun, 2004: Effects of environmentally induced asymmetries on hurricane intensity: A numerical study. J. Atmos. Sci., 61, 3065-3081, https://doi.org/10.1175/JAS-3343.1.

Yano, J.-I., and K. A. Emanuel, 1991: An improved model of the equatorial troposphere and its coupling with the stratosphere. J. Atmos. Sci., 48, 377-389, https://doi.org/10.1175/ 1520-0469(1991)048<0377:AIMOTE > 2.0.CO;2.

Young, I. R., 2017: A review of parametric descriptions of tropical cyclone wind-wave generation. Atmosphere, 8, 194, https:// doi.org/10.3390/atmos8100194.

Zeng, Z., Y. Wang, and C.-C. Wu, 2007: Environmental dynamical control of tropical cyclone intensity-An observational study. Mon. Wea. Rev., 135, 38-59, https://doi.org/10.1175/MWR3278.1.

Zhang, F., and K. Emanuel, 2016: On the role of surface fluxes and WISHE in tropical cyclone intensification. J. Atmos. Sci., $\mathbf{7 3}$, 2011-2019, https://doi.org/10.1175/JAS-D-16-0011.1.

Zhang, L., and L. Oey, 2018: An observational analysis of ocean surface waves in tropical cyclones in the western North Pacific Ocean. J. Geophys. Res. Oceans, https://doi.org/10.1029/ 2018JC014517, in press. 